\title{
Estimación de la volatilidad del tipo de cambio en México y Brasil. Un enfoque con modelos Markov Switching Garch
}

\section{Estimation of Volatility of the Rate of Exchange in Mexico and Brazil. An Approach with Markov Switching Garch Models}

Rolando Caballero Martínez* Benigno Caballero Claure**

\section{Resumen}

Este documento analiza la evolución de la volatilidad cambiaria en México y Brasil en el periodo 1994-2014, y presenta evidencia de que la misma tiende a disminuir con el tiempo. Además, se analiza la relación existente entre la volatilidad cambiaria y la tasa de depreciación. Nuestros hallazgos indican que tres de cinco modelos de volatilidad condicional confirman que una mayor depreciación cambiaria precede temporalmente a una mayor volatilidad cambiaria. Asimismo, para poder analizar estos efectos se emplean modelos de heterocedasticidad condicional (ARCH-M, GARCH-M, TGARCH-M, EGARCH-M, y PARCH-M), así como modelos markovianos: Switching Markov Regression. Los resultados demuestran que, una vez que la volatilidad se encuentra en un determinado régimen (alto o bajo), es muy baja la probabilidad de que pase al otro régimen de manera inmediata. Otro hallazgo importante es

\footnotetext{
* Facultad de Economía, Universidad Nacional Autónoma de México (UNAM), Ciudad de México, México. Contacto: roland.caballerom@comunidad.unam.mx.

** Facultad de Economía, Universidad Técnica de Oruro (UTO), Oruro, Bolivia.

Contacto: b_caballero_c@hotmail.com.
} 
la persistencia alta en volatilidad en ambas economías, lo que confirmaría que los choques en ella no se disipan rápidamente.

Palabras clave: Volatilidad estocástica; econometría financiera; modelos EGARCH; volatilidad estocástica, econometría financiera; modelos EGARCH.

\section{Abstract}

This paper analyzes the evolution of exchange rate volatility in Mexico and Brasil in the period 1992:01-2013:12 and presents evidence that it tends to decrease over time. We also discuss the relationship between exchange rate volatility and depreciation. Our findings indicate that further depreciation change temporally precedes greater exchange rate volatility. Also to analyze these effects models conditional heteroskedasticity (ARCH-M, GARCH-M, TGARCH-M, EGARCH-M and PARCH-M) was used. The results of our study show that once the volatility is in a regime is very low probability of passing to another regime immediately. Another important finding is the high persistence in volatility in both economies, confirming that shocks it cannot dissipate quickly.

Keywords: Stochastic Volatility, Financial Econometrics, EGARCH-M

Clasificación/Classification JEL: C01, C58, E47

\section{Introducción}

A menudo las variaciones del tipo de cambio y una elevada volatilidad cambiaria son consideradas como un mecanismo de contagio de crisis internacionales hacia la economía nacional, y ello se agudiza más si cumple tres rasgos característicos. Primero, si el país tiene una fuerte apertura comercial; segundo, si tiene un elevado traspaso del tipo de cambio hacia los precios domésticos; y por último, si tiene deudas predominantemente dolarizadas. Esto es así debido a que una elevada volatilidad cambiaria termina por ir en desmedro de la población consumidora de bienes importados y de empresas que utilizan en su proceso productivo gran porcentaje de insumos externos. Asimismo, con frecuencia el tipo de cambio es utilizado por los corredores de bolsa e inversionistas financieros para cuantificar riesgos, y de ahí su relevancia; por lo tanto, cuanto mayor es la volatilidad, mayor es la variabilidad del tipo de cambio y, por lo mismo, mayor el riesgo. 
Por otra parte, las crisis económicas internacionales que se han dado en los últimos años y que tuvieron como génesis a Europa y Estados Unidos, y la fuerte relación comercial y financiera que México y Brasil tienen con Estados Unidos y Europa, han tenido efectos importantes en el comportamiento del tipo de cambio nominal en ambos países. Por lo mismo, esta dependencia económica y las crisis internacionales han dado lugar en las últimas décadas a depreciaciones/apreciaciones cambiarias en México y Brasil, respectivamente, que han menoscabado las expectativas de estabilidad y certidumbre.

El objetivo de este documento de investigación consiste en analizar y estimar una medida de volatilidad cambiaria para México en el periodo 1996m01-2013m12 y Brasil para el periodo $1994 \mathrm{~m} 07-2014 \mathrm{~m} 08$, con datos mensuales, toda vez que la volatilidad cambiaria sigue siendo importante para cualquier actividad económica que implique intercambios internacionales, sean de activos físicos y/o financieros. Asimismo, el periodo de estudio es interesante, ya que involucra periodos de alta volatilidad cambiaria acompanados de crisis económicas y financieras a nivel mundial. Todo lo anterior a partir de un análisis del comportamiento mensual del tipo de cambio FIX publicado por el Banco de México (Banxico) y el Banco Central del Brasil.

En la actualidad se dispone de diferentes metodologías para la estimación de la volatilidad. La más sencilla y ampliamente utilizada es la medida a través de la desviación estándar de los datos, conocida como volatilidad histórica o clásica. A la vez, existen modelos que se basan en los precios intradía (máximos y mínimos), y otros modelos no lineales, como los procesos ARCH, GARCH, TGARCH, EGARCH y PARCH, que son medidas más formales para estimar la volatilidad. Y por último, se cuenta con los modelos de volatilidad estocástica con cambio de régimen Switching Regression Model. En el presente trabajo de investigación se utilizarán las dos últimas metodologías econométricas.

Algunos estudios de la utilización de los modelos de las familias ARCH para estimar la volatilidad estocástica de una determinada variable, en especial del tipo de cambio, son, por ejemplo, del Nobel de Economía 2003 Robert Engle (1982), que realiza una estimación de la volatilidad de la inflación para el Reino Unido utilizando modelos de heterocedasticidad condicional ARCH. Asimismo, Sengupta y Sfeir (1996) estudian la volatilidad cambiaria del dólar estadounidense respecto de la libra esterlina, el yen, el marco alemán y el franco francés, utilizando como herramientas modelos GARCH; Tse (1998) estudia la volatilidad del tipo de cambio yen-dólar utilizando modelos GARCH; Domowitz y Hakkio (1985) aplican un modelo GARCH-M para detectar primas de riesgo en monedas europeas, no encontrando 
evidencia a favor de las mismas; Sandoval (2006) analiza y estima la volatilidad cambiaria de Brasil, Colombia, Corea del Sur, Chile, México, Tailandia e India, que son países considerados emergentes. Por último, tenemos el estudio de Sánchezy Reyes (2006) para el caso mexicano, cuyo trabajo enfatiza el papel que han jugado las regularidades empíricas en el desarrollo de modelos de volatilidad y argumentan que los modelos GARCH han sido exitosos porque permiten capturar regularidades empíricas en las series económico-financieras.

Ahora, tomando como base la evidencia empírica de los modelos de las familias GARCH aplicados al estudio del tipo de cambio en economías emergentes y desarrolladas, la clave de estos modelos, según Engle y Bollerslev (1986), está en considerar la información pasada de la variable y su volatilidad observada como factor altamente explicativo de su comportamiento presente y, por extensión lógica, de su futuro predecible. Estadísticamente, esta conclusión se refleja en tener en cuenta la esperanza condicional (conocida y fija la información hasta el momento inmediatamente anterior) del cuadrado de una variable (la expresión de su varianza si su media es nula). En síntesis, los modelos de las familias GARCH no solamente permiten hacer un análisis y estimación de la volatilidad cambiaria, sino también realizar pronósticos de la volatilidad para el periodo siguiente, y por esa razón es que nosotros utilizamos de manera inicial esta metodología econométrica, para luego complementar nuestro análisis de la volatilidad cambiaria con los modelos estocásticos de cambio de régimen (Switching Regression Model) ${ }^{1}$, propuesto inicialmente por Hamilton (1989).

En esa línea, la ventaja que tienen los modelos con cambio de régimen se debe a que, en un entorno de volatilidad, es imprescindible no solo conformarse con estimaciones de la volatilidad y su respectivo pronóstico, sino que es de gran preponderancia para los agentes económicos e inversores financieros contar con información respecto a las probabilidades que tienen de observar baja o alta volatilidad cambiaria, la persistencia de las mismas y la probable duración de cada uno de los estados, es decir, cuánto se espera que dure un periodo de alta (baja) volatilidad y cuál es la probabilidad de que se pase a un estado de alta volatilidad cuando el tipo de cambio se encuentra en el estado de baja volatilidad o viceversa. Sin embargo, las probabilidades de estar en un régimen de baja o alta volatilidad cambiaria, así como su persistencia, no son directamente observables y, por lo mismo, deben inferirse a partir de la información disponible para los agentes económicos. Es por ese motivo que nosotros hacemos hincapié en los modelos con cambio de régimen, ya que este considera

1 Todas las metodologías se aplican con la finalidad de analizar y estimar la volatilidad cambiaria y evaluar si la misma tiende a aumentar o disminuir con el paso del tiempo. 
los cambios de estado de la volatilidad cambiaria y además nos permite dar respuesta a lo enunciado en líneas anteriores.

Almismo tiempo, esta metodología consiste en caracterizarla evolución de una variable por un proceso de media condicionada a un estado de la naturaleza específico, con su consiguiente volatilidad, lo cual permite que esta variable salte en nivel a procesos de crecimiento alternativos, con medias y volatilidades particulares, representando finalmente un proceso de tendencia estocástica. Un producto de esta metodología consiste en la determinación de las probabilidades condicionales de estar en cada estado de la naturaleza predefinido, de manera de poder determinar con certeza en qué estado de la naturaleza estamos en un período de tiempo específico, lo cual facilita una evaluación ex-post de la política económica. Trabajos empíricos que utilizan modelos dinámicos de cambios de régimen que siguen una cadena de Markov (Markov Switchingo MS) son los de Engel y Hakkio (1994) y GómezPuig y Montalvo (1997); emplean las probabilidades filtradas que se obtienen con este tipo de modelos para categorizar los regímenes de calma y crisis cambiaria experimentados por economías pertenecientes al Sistema Monetario Europeo a inicios de los 90’s, logrando así una mejor caracterización respecto a metodologías anteriores.

El artículo está estructurado en cuatro apartados. En el primero se realiza una descripción breve del tema. En el segundo se describe la metodología econométrica. En el tercero se describen los resultados de estimación y evaluación de pronósticos, y en el último se muestran las conclusiones finales de nuestros resultados.

\section{Metodología econométrica}

La hipótesis de varianza constante de muchas series económicas y financieras se ajusta poco a la realidad. La familia de modelos ARCH y GARCH pretende resolver este problema, pues constituye un intento de conseguir predictores de esa volatilidad utilizando información condicional, en este caso información sobre la estructura del término de error, que permita llevar a cabo una estimación de la volatilidad de la variable en estudio durante períodos más cortos. 


\subsection{Modelos de las familias GARCH}

En este apartado daremos a conocer de manera breve los modelos de las familias GARCH que utilizaremos en nuestro trabajo de investigación. Asimismo, respecto a la utilidad y empleo de los modelos ARCH, existen, por lo menos, dos recopilaciones muy significativas (Bollerslev y otros, 1992 y 1994. En ellas se citan más de cuatrocientas aplicaciones diferentes que se han producido en los primeros doce años de existencia de este tipo de modelos. Con frecuencia, las aplicaciones se han centrado en el campo de la economía financiera y, más concretamente, en la aplicación de teorías de valoración de riesgos en la construcción de carteras de inversión a partir de una conveniente modelización de la volatilidad o varianza de una determinada variable.

Con respecto al modelo 1 del Cuadro 1, el premio Nobel de economía Robert Engle (1982) realiza una estimación de la volatilidad de la inflación para el Reino Unido, utilizando modelos de heterocedasticidad condicional ARCH. Con relación a los modelos GARCH (modelo 2), son capaces de recoger los agrupamientos de volatilidad que se observan en las series de rendimientos financieros, pero no son útiles para captar comportamientos asimétricos ante innovaciones de carácter positivo o negativo. En cuanto a los modelos TGARCH (modelo 3), podemos afirmar que una desventaja que presenta la especificación GARCH es que impone simetría en los shocks de depreciación cambiaria. El coeficiente del término ARCH $\left(\alpha_{1}\right)$ del Modelo GARCH $(1,1)$ del modelo 2, recoge los efectos de los shocks, sin distinguir entre el signo de los mismos. Si la varianza condicional de los errores responde de manera distinta a shocks negativos y positivos, se estaría incurriendo en un error de especificación en la especificación GARCH (1,1). El modelo EGARCH (modelo 4), que utilizamos en nuestras estimaciones, tiene diversas ventajas sobre los modelos ARCH y GARCH tradicionales. Primero, permite capturar asimetrías en la respuesta de la volatilidad cambiaria a los shocks de depreciación. Segundo, a diferencia de los modelos GARCH, el modelo EGARCH, especificado en logaritmos, no impone restricciones de no negatividad sobre los parámetros. Finalmente, modelizar la volatilidad cambiaria en logaritmo disminuye el efecto de outlier (observaciones anormales o aberrantes) sobre los resultados de la estimación. 


\section{Cuadro 1}

\section{Modelos econométricos de las familias GARCH}

\begin{tabular}{|c|c|c|c|}
\hline Modelo & $\begin{array}{l}\text { Año } \\
\text { Autor }\end{array}$ & Aportación principal & Especificación del modelo \\
\hline $\begin{array}{l}\text { Modelo (1) } \\
\text { ARCH }\end{array}$ & Engle & $\begin{array}{l}\text { Primera especificación y } \\
\text { desarrollo. }\end{array}$ & $\begin{array}{l}\xi_{t}=\sigma_{t} \xi_{t} \\
\sigma_{t}^{2}=\alpha_{0}+\alpha_{1} \varepsilon_{t-1}^{2}+\alpha_{m} \varepsilon_{t-m}^{2}\end{array}$ \\
\hline $\begin{array}{l}\text { Modelo (2) } \\
\text { GARCH }(1,1)\end{array}$ & $\begin{array}{l}\text { Bollerslev y } \\
\text { Taylor }\end{array}$ & $\begin{array}{l}\text { Método generalizado } \\
\text { sin restricciones para } \\
\text { la estimación de los } \\
\text { parámetros ARCH con } \\
\text { infinitos retardos. }\end{array}$ & $\begin{array}{l}e_{t}=\sum_{t \varepsilon J} \rho_{j} e_{t-j} \\
\varepsilon_{t} \approx\left(0, \sigma_{t}^{2}\right) \\
\sigma_{t}^{2}=\alpha_{0}+\alpha_{1} \varepsilon_{t-1}^{2}+\gamma_{1} \sigma_{t-1}^{2}\end{array}$ \\
\hline $\begin{array}{l}\text { Modelo (3) } \\
\operatorname{TGARCH}(1,1)\end{array}$ & $\begin{array}{l}\text { Glosten, } \\
\text { Jagannathan } \\
\text { y Runkle }\end{array}$ & $\begin{array}{l}\text { Carácter asimétrico de } \\
\text { la respuesta a shocks } \\
\text { positivos o negativos. }\end{array}$ & $\begin{array}{l}\sigma_{t}^{2}=\alpha_{0}+\sum_{i=1}^{m} \alpha_{i} \varepsilon_{t-i}^{2}+\sum_{i=1}^{m} \gamma_{i} \varepsilon_{t-i}^{2} d_{t-i}+\sum_{j=1}^{s} \beta_{j} \sigma_{t-j}^{2} \\
\text { Donde: } \\
\qquad \mathrm{I}_{i t}=\left\{\begin{array}{lll}1 & \varepsilon_{t-1}<0 \\
0 & \varepsilon_{t-i}>0\end{array}\right\}\end{array}$ \\
\hline $\begin{array}{l}\text { Modelo (4) } \\
\text { EGARCH }\end{array}$ & Nelson & $\begin{array}{l}\text { Modelos ARCH para } \\
\text { procesos no normales } \\
\text { (funciones de densidad } \\
\text { exponenciales). Carácter } \\
\text { asimétrico de la respuesta } \\
\text { a shocks positivos o } \\
\text { negativos. }\end{array}$ & $\begin{array}{l}e_{t}=\sum_{j \varepsilon, J} \rho_{j} e_{t-j}+\varepsilon_{t} \\
\varepsilon_{t} \approx\left(0, \sigma_{t}^{2}\right) \\
\ln \sigma_{i t}^{2}=\beta_{0}+\beta_{1} \ln \sigma_{t-1}^{2}+\gamma_{1} \frac{\varepsilon_{t-1}}{\sigma_{t-1}^{2}}+\varphi_{1} \frac{\left|\varepsilon_{t-1}\right|}{\left|\sigma_{t-1}^{2}\right|} \\
\left|\beta_{1}\right|<1\end{array}$ \\
\hline $\begin{array}{l}\text { Modelo (5) } \\
\text { PARCH }\end{array}$ & Ding et al. & $\begin{array}{l}\text { Se propone modelizar } \\
\text { un valor potencial de } \\
\text { la desviación típica } \\
\text { que atienda al máximo } \\
\text { de la función de } \\
\text { autocorrelación del valor } \\
\text { absoluto del proceso }\end{array}$ & $\sigma_{t}^{\delta}=\omega+\sum_{j=1}^{q} \beta_{j} \sigma_{t-j}^{\delta}+\sum_{i=1}^{p}\left\{\alpha_{i}\left|\varepsilon_{t-j}\right|-\gamma_{i} \varepsilon_{t-j}\right\}^{\delta}$ \\
\hline
\end{tabular}

Fuente: elaboración propia de los autores

Por último, los modelos PARCH (modelo 5) modelizan las potencias de la desviación típica. Originalmente fue propuesto por Taylor (1986) y Schwert (1989) para modelizar las desviaciones típicas, y posteriormente fue generalizado por Ding et al. (1993).

\subsection{Modelos Switching Regression}

El modelo de cambio de régimen fue desarrollado por Hamilton (1989); con probabilidad de transición constante, es uno de los más populares para tratar con modelos no lineales de series de tiempo. Sin embargo, diferentes consideraciones económicas sugieren el deseo de permitir que las probabilidades de transición sean variables. Diebold et al. (1993) proponen 
una clase de modelos de cambio de Markov, en los cuales la probabilidad de transición de régimen es endógena ${ }^{2}$, variables en el tiempo, es decir donde puedan variar con los fundamentos económicos y/o otras variables exógenas.

\subsubsection{Supuestos en el análisis}

Existen tres supuestos en la aplicación del modelo de cambio de régimen de Markov utilizados en este trabajo. El primero es que existen dos estados: período de alta volatilidad y período de baja volatilidad cambiaria. Dado que los estados son no observables directamente, éstos son representados por una variable binaria $\left(S_{t}\right)$, la cual está latente. El segundo implica que existen variables directamente observables cuyos cambios de comportamiento está influido por el valor de la variable $S_{t}$. El comportamiento del tipo de cambio es diferente durante períodos de presiones especulativas que durante períodos de relativa calma. En particular, en teoría se espera una mayor volatilidad del tipo de cambio y una mayor tasa de depreciación durante ataques especulativos. Finalmente, se asume que, dado el estado actual de la variable $S_{t}$-alta y baja volatilidad- existe una probabilidad cierta de permanecer en el mismo estado, o de moverse hacia el otro estado. En el modelo teórico, la probabilidad de moverse de un estado de baja volatilidad hacia uno de alta volatilidad y/o viceversa, depende del estado de ciertos fundamentos económicos y de variables de economía política.

\subsubsection{El modelo}

La variable latente en el modelo sigue una cadena de Markov de 2 estados de primer orden, donde $S_{t}=1$ (estado volatilidad baja) y $S_{t}=2$ (estado volatilidad alta). Cuando el proceso está en el régimen 1 , se presume que la variable observada $y_{t}$-que en nuestro caso es la primera diferencia logarítmica del tipo cambio nominal de venta- se puede representar por una distribución $N\left(\mu_{1}, \sigma_{1}^{2}\right)$. Si el proceso está en el régimen $2, y_{t}$ se representa por una distribución $N\left(\mu_{2}, \sigma_{2}^{2}\right)$. Por lo tanto, el comportamiento de la variable dependiente $y_{t}$ es dependiente de $S_{t^{\prime}}$ de forma que:

$$
\frac{y_{t}}{s_{t}} \approx N\left(\mu_{i}, \sigma_{i}^{2}\right)
$$

La densidad de $\mathrm{y}_{\mathrm{t}}$ condicional a $\mathrm{S}_{\mathrm{t}}$ es:

2 El primer trabajo en esta área es de Lee (1991). 


$$
f\left(y_{t} / s_{t}\right)=1 / \sqrt{2 \pi} \sigma_{s_{t}} \exp \left(\frac{-\left(y_{t}-\mu_{s_{t}}\right)^{2}}{2 \sigma_{s_{t}}^{2}}\right) \text { para } \mathrm{S}_{\mathrm{t}}=1,2
$$

La variable latente del cambio de régimen $S_{t}$ se caracteriza de acuerdo a la siguiente matriz de probabilidad $\mathrm{P}_{\mathrm{t}}$ :

\section{Momento $t$}

\section{Estado 1}

Momento $t-1\left[\begin{array}{lll}\text { Estado } 1 & p_{11}^{t}=F\left(x_{t-1}^{\prime} \beta_{1}\right) & p_{12}^{t}=1-F\left(x_{t-1}^{\prime} \beta_{1}\right) \\ \text { Estado 2 } & p_{21}^{t}=1-F\left(x_{t-1}^{\prime} \beta_{2}\right) & p_{22}^{t}=F\left(x_{t-1}^{\prime} \beta_{2}\right)\end{array}\right]$

donde $p_{i j}$ es la probabilidad de pasar del estado i en período $t-1$ al estado j en el período t, y F es una función de distribución acumulativa normal. Los elementos del vector $(k x 1) x_{t-1}$ son las variables que afectan la probabilidad de transición $\beta$ son parámetros estado dependientes y que deberán ser estimados.

Para completar el modelo, es necesario el valor inicial de la probabilidad incondicional de estar en el estado 1 en el momento $1 P\left(S_{t}=1\right)$. El procedimiento de estimación utilizado es máxima verosimilitud, donde la función de verosimilitud se calcula utilizando la iteración descrita en Hamilton (1994:692-3).

Se presume que el régimen no observable $\left\{S_{\mathrm{t}}\right\}$ ha sido generado por alguna distribución de probabilidad, para la cual la probabilidad incondicional de que $S_{t}$ tome el valor $j$ se denota por $\pi_{i}$ :

$$
P\left\{s_{t}=j ; \theta\right\}=\pi_{j} \quad \text { para } j=1,2, \ldots, N
$$

Las probabilidades $\pi_{1}, \ldots, \pi_{\mathrm{N}}$ son incluidas también en $\theta$; esto significa que $\theta$ viene dado por:

$$
\theta=\left(\mu_{1}, \ldots, \mu_{N} ; \sigma_{1}^{2}, \ldots, \sigma_{N}^{2} ; \pi_{1}, \ldots, \pi_{N}\right)^{\prime \prime}
$$


La función de densidad conjunta de $y_{t}$ y $S_{t}$

$$
\begin{gathered}
p\left(y_{t}, s_{t} ; \theta\right)=f\left(y_{t} / s_{t}=j ; \theta\right) \cdot P\left\{s_{t}=j ; \theta\right\} \\
p\left(y_{t}, s_{t}=j ; \theta\right)=\pi_{j} / \sqrt{2 \pi} \sigma_{j} \exp \left\{-\left[y_{t}-\mu_{j}\right]^{2} / 2 \sigma_{j}^{2}\right\}
\end{gathered}
$$

La distribución incondicional de $y$ puede obtenerse al sumar la ecuación anterior sobre todos los valores de j:

$$
f\left(y_{t}: \theta\right)=\sum_{j=1}^{N} p\left(y_{t}, s_{t}=j ; \theta\right)
$$

Dado que el régimen $S$ es no observable, la expresión anterior es la densidad relevante que describe el dato observado actual $y_{t}$ Si la variable régimen $S_{t}$ se distribuye i.i.d. en diferentes momentos $t$, el logaritmo de la verosimilitud para los datos observados puede ser calculado de la distribución incondicional como,

$$
\ell(\theta)=\sum_{t=1}^{T} \log f\left(y_{t} ; \theta\right)
$$

La estimación de máxima verosimilitud de $\theta$ se obtiene maximizando la ecuación anterior sujeta a las restricciones de que $\pi_{1}+\ldots+\pi_{N}=1$ y que $\pi_{j} \geq 0$ para $j=1,2, \ldots, N$.

Una vez obtenidas las estimaciones de $\theta$, es posible realizar una inferencia sobre qué régimen es más probable que sea el responsable de producir la observación $t$ de $y_{t}$.

De la definición de probabilidad condicional se sigue que:

$$
P\left\{s_{t}=j / \mathrm{y}_{\mathrm{t}} ; \theta\right\}=\frac{p\left(y_{t}, s_{t} ; \theta\right)}{f\left(y_{t} ; \theta\right)}=\left\{\pi_{j} f\left(y_{t} / s_{t}=j ; \theta\right) / f\left(y_{t} ; \theta\right)\right\}
$$

Este número representa la probabilidad, dado los datos observados, de que el régimen no observable de la observación t sea el régimen $j$. 
Dado que las ecuaciones son no lineales, no es posible resolver analíticamente la estimación de $\theta$ como función de $\left\{y_{1}, y_{2}, \ldots, y_{T}\right\}$. Sin embargo, esas ecuaciones sugieren recurrir a un algoritmo iterativo para encontrar el estimador máximo verosímil. Empezando por un valor inicial arbitrario de búsqueda de $\theta$, se comienza iterando hasta que el cambio entre dos estimaciones es menor que algún criterio de convergencia especificado.

\section{Metodología y resultados de la estimación}

La hipótesis de varianza constante de muchas series económicas y financieras se ajusta poco a la realidad. La familia de modelos $\mathrm{ARCH}$ y GARCH pretende resolver este problema, y constituye un intento de conseguir predictores de esa volatilidad utilizando información condicional, en este caso información sobre la estructura del término de error, que permita llevar a cabo una estimación de la volatilidad de la variable en estudio durante períodos más cortos. En esa línea, el objetivo es analizar y estimar una medida de volatilidad del tipo de cambio peso-dólar y real-dólar para México y Brasil, respectivamente.

\subsection{Resultados de la estimación}

Se combinarán las metodologías SARIMA (Autoregressive Integrated Moving Average) ${ }^{3}$ y GARCH (General Autoregressive Conditional Heteroskedasticity) tomando como proxy de la tasa de depreciación cambiaria, la variación mensual del tipo de cambio nominal pesodólar y real-dólar, con información mensual. De este modo se puede estimar tanto la media condicional como la varianza condicional, a través de métodos de máxima verosimilitud. La modelación de la serie de tasa de depreciación cambiaria inicialmente se la realiza a través de un proceso SARIMA, que permite predecir la tasa de depreciación en cada periodo, basándose en la información contenida en la serie en los periodos anteriores, es decir, en los rezagos de la serie y capturando la estacionalidad de la misma. Así, se obtienen los errores de predicción para cada periodo. La utilización de los modelos de las familias ARCH, por su parte, permite obtener una serie temporal de la varianza de los errores que sirve como aproximación de la volatilidad cambiaria.

3 Los modelos ARIMA son parte de la metodología habitual de series de tiempo y permiten estudiar el comportamiento de una variable aleatoria a través del tiempo, utilizando sólo la información contenida en la serie histórica de la propia variable. La forma genérica de un modelo ARIMA para una variable X se escribe en la terminología habitual de Box y Jenkins. 
Comprobado el comportamiento estacionario de la variación mensual del tipo de cambio nominal peso-dólar y real-dólar, que resulta ser una proxy de la tasa de depreciación, se estima el modelo SARIMA para esa variable. Utilizando el paquete estadístico TRAMOSEATS se identificó un modelo SARIMA para México $(0,1,1)(1,1,1)$, con lo cual el modelo identifica una diferenciación y un parámetro de media móvil en la parte regular, así como una diferenciación, un parámetro autorregresivo y de medias móviles en la parte estacional. Para Brasil también se identificó un modelo SARIMA $(0,1,1)(1,1,1)$, con lo cual el modelo identifica una diferenciación y un parámetro de media móvil en la parte regular, así como una diferenciación, un parámetro autorregresivo y de medias móviles en la parte estacional. El modelo identifica igualmente una serie (outliers) de diferente índole (impulso o escalón), a partir de lo cual se realizó un calibramiento del mismo, con la finalidad de mejorar el modelo inicial para el caso de Brasil. Los mejores resultados para el caso de Brasil se obtuvieron para un modelo con una diferenciación tanto en la parte regular como en la estacional, un componente $\mathrm{AR}(7), \mathrm{MA}(1)$ en la parte regular y un componente SMA(1) Y SAR(1) en la parte estacional.

La estimación del modelo se presenta en el Cuadro 1, para México, y Cuadro 2, para Brasil; entre otras cosas, se puede advertir en ambos modelos una alta significancia de las variables, ausencia de correlación en los residuos, estabilidad del modelo SARIMA y no normalidad en los residuos. Sin embargo, se requiere verificar si la varianza residual de la tasa de depreciación es constante en el tiempo, para lo cual se incluye en los cuadros el estadístico para la prueba de efectos $\mathrm{ARCH}$, que evalúa si la varianza de los residuos, $\varepsilon_{\mathfrak{t}^{\prime}}$ es constante. La prueba indica que se rechace la hipótesis nula de existencia de un proceso con varianza constante para México y Brasil, en vista de lo cual se puede modelar el proceso de varianza condicional (volatilidad cambiaria) para los residuos, por medio de los modelos autorregresivos de heterocedasticidad condicional ARCH (Sánchez y Reyes, 2006). 


\section{Cuadro 2}

Modelo Sarima para la tasa de depreciación cambiaria en México

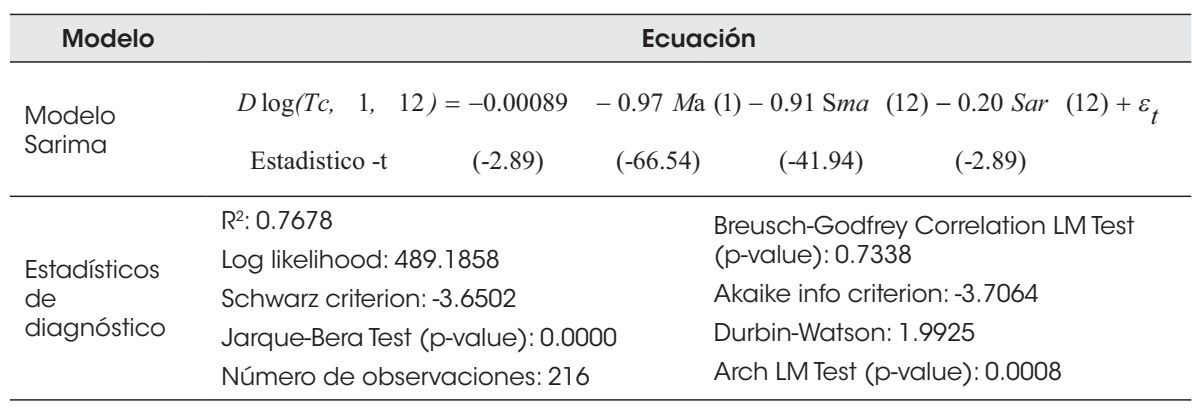

Fuente: elaboración propia de los autores

Cuadro 3

Modelo Sarima para la tasa de depreciación cambiaria en Brasil

\begin{tabular}{|c|c|c|}
\hline Modelo & & Ecuación \\
\hline $\begin{array}{l}\text { Modelo } \\
\text { Sarima }\end{array}$ & $\begin{array}{c}D \log (T c, \quad 1, \quad 12)=-0.00016 \quad-0.18 \mathrm{Ar} \\
\text { Estadistico -t } \quad(-2.21) \quad(-2.26)\end{array}$ & $\begin{array}{c}\text { (7) }-0.84 \text { Ma }(1)-0.09 \text { Sar }(12)-0.90 \text { Sma }(12)+\varepsilon_{t} \\
(-17.85) \quad(-1.07)\end{array}$ \\
\hline $\begin{array}{l}\text { Estadísticos } \\
\text { de } \\
\text { diagnóstico }\end{array}$ & $\begin{array}{l}R^{2}: 0.6717 \\
\text { Log likelihood: } 332.824 \\
\text { Schwarz criterion: }-2.8380 \\
\text { Jarque-Bera Test (p-value): } 0.0000 \\
\text { Número de observaciones: } 242\end{array}$ & $\begin{array}{l}\text { Breusch-Godfrey Correlation LM Test } \\
\text { (p-value): } 0.9526 \\
\text { Akaike info criterion: }-2.9139 \\
\text { Durbin-Watson: } 1.9246 \\
\text { Arch LM Test (p-value): } 0.0087\end{array}$ \\
\hline
\end{tabular}

Fuente: elaboración propia de los autores

Asimismo, en el Gráfico 3 se muestran los correlogramas. Por otro lado, el Gráfico 4 muestra las funciones impulso-respuesta para México y Brasil, que en un modelo ARMA, correctamente especificado, teóricamente debería esperarse que la respuesta ante una innovación desaparezca en forma asintótica, es decir que tienda a cero en un horizonte de corto plazo; éste es un indicador de que el modelo es estacionario. Por otra parte, el impulsorespuesta acumulado deberá tender al valor de largo plazo de la variable que se está modelando, también en un horizonte de corto plazo.

Asimismo, con respecto a los gráficos 1 y 2, calculan y presentan el espectro de la variable residuos del modelo SARIMA para México y Brasil. Sin la opción (Bartlett) se ofrece el periodograma muestral. Con dicha opción, se utiliza una ventana de retardos de Barlett de longitud $2(\mathrm{~T})^{\wedge} 0.5$ (donde $\mathrm{T}$ es el tamaño muestral para estimar el espectro). Ahora bien, 
cuando se presenta el periodograma muestral, también se proporciona una contraste $t$ sobre integración fraccional de la serie "residuos del SARIMA" (memoria larga), donde la hipótesis nula es que el orden de integración es cero.

\section{Gráfico 1: Periodograma de los residuales del modelo SARIMA (arriba) y ventana de espectros de Bartlett (abajo) para México}

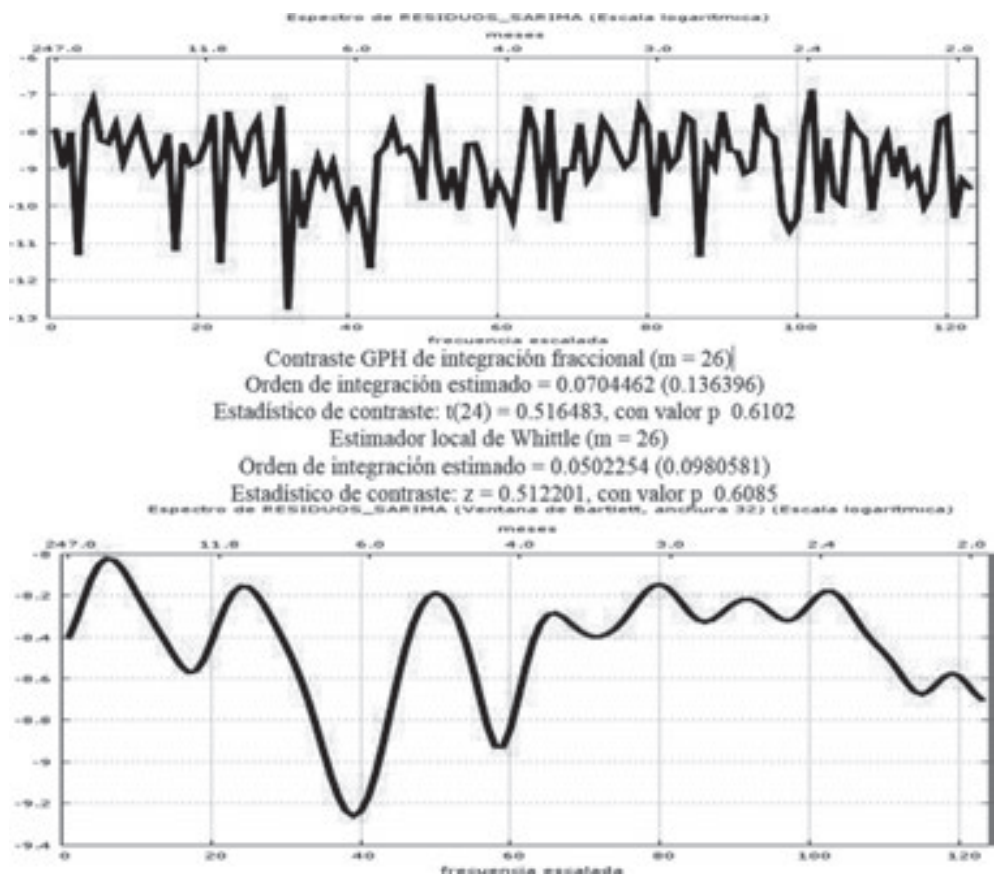

Fuente: elaboración propia de los autores 
Gráfico 2: Periodograma de los residuales del modelo SARIMA (arriba) y ventana de espectros de Bartlett (abajo) para Brasil

Espectro de RESDUOS_SARIM (Escala logaritmica)

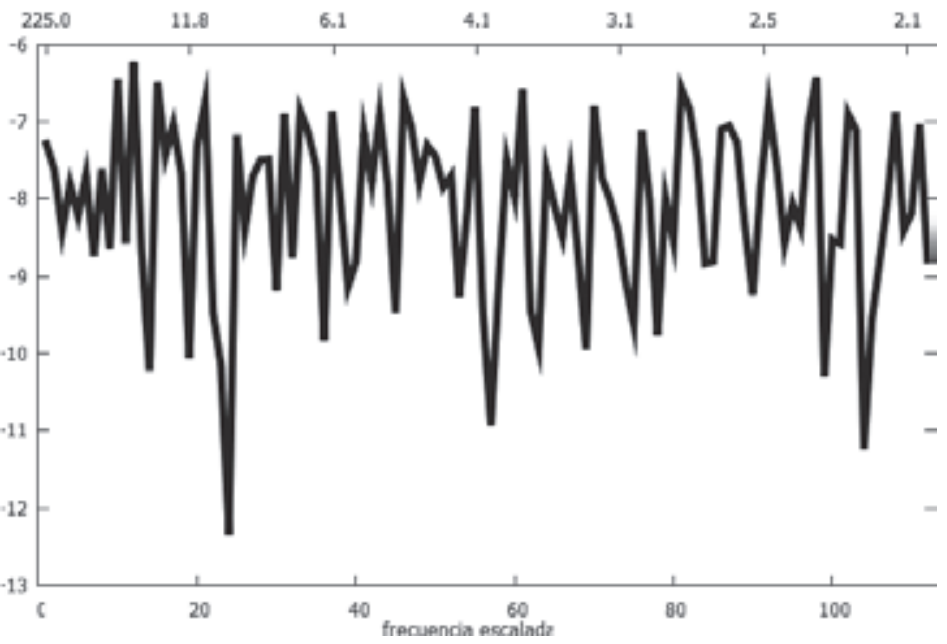

Contraste GPH de integración fraccional $(m=25)$

Orden de integración estimado $=0.198094(0.174784)$

Estadístico de contraste: $\dagger(23)=1.13337$, con valor $\mathrm{p} 0.2687$

Estimador local de White $(m=25)$

Orden de integración estimado $=-0.0218394(0.1)$

Estadístico de contraste: $z=-0.218394$, con valor $\mathrm{p} 0.8271$

[spectro de RCSIDUOS_SARDMA (Ventana de Bartiett, anchura 30) (Cscala logaritmika)

meses

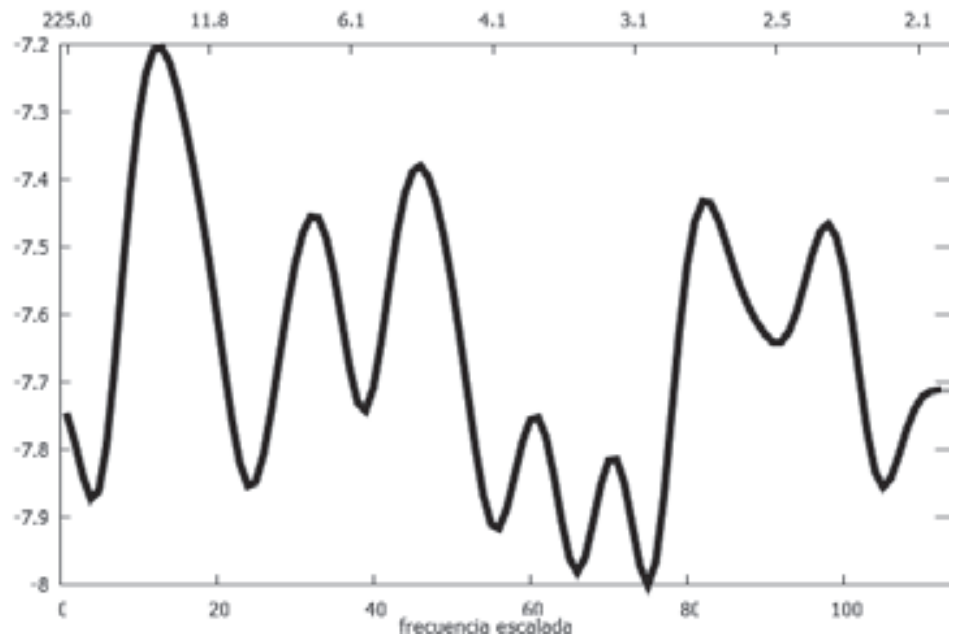


Gráfico 3: Correlograma del modelo Sarima México
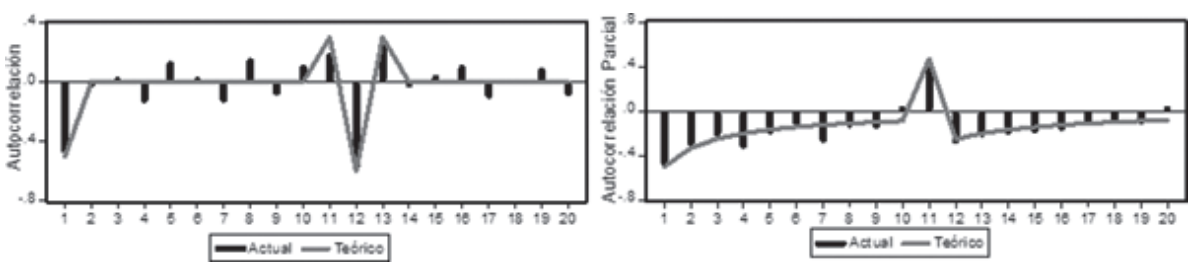

Correlograma del modelo Sarima Brasil

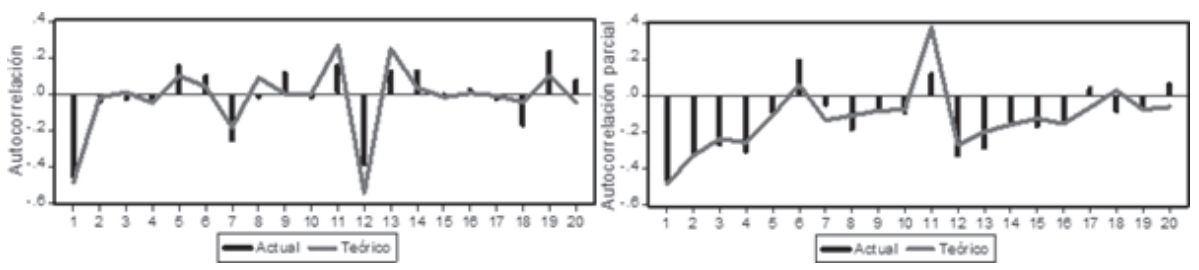

Fuente: elaboración propia de los autores

Gráfico 4: Estabilidad del Modelo SARIMA (FIR acumulado y sin acumular) para México
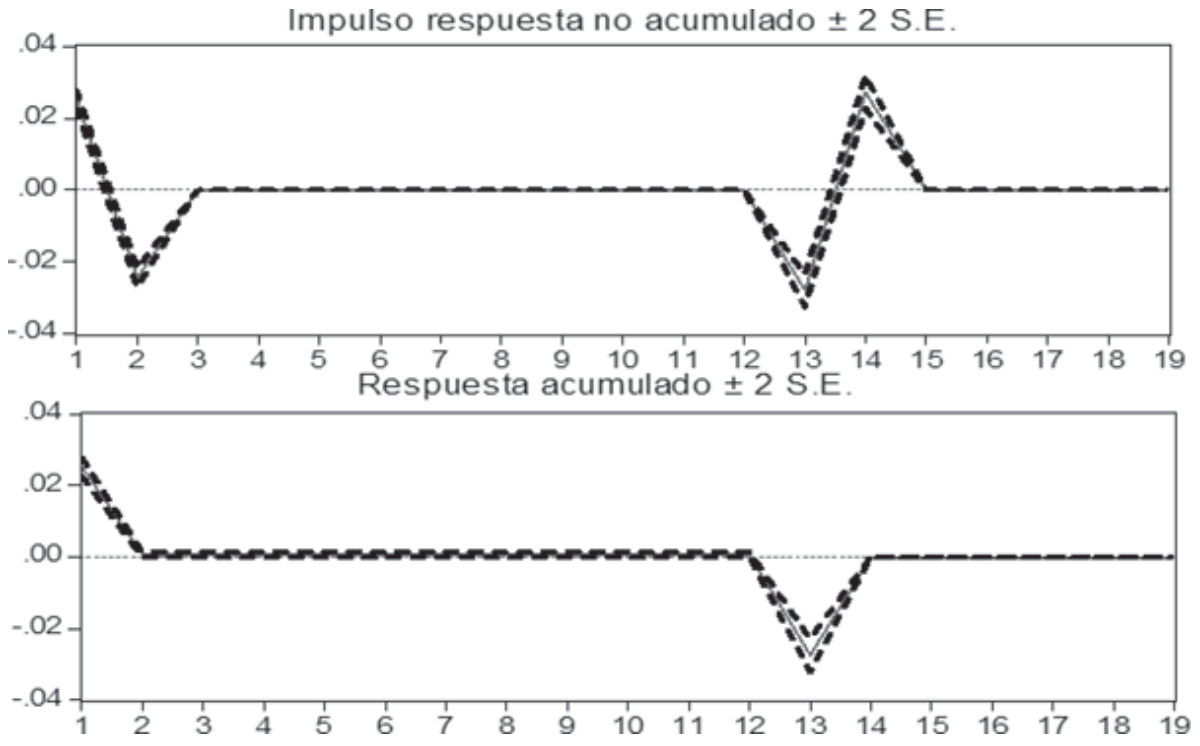
Estabilidad del Modelo SARIMA (FIR acumulado y sin acumular) para Brasil Impulso respuesta no acumulado $\pm 2 \mathrm{~S}$.E.
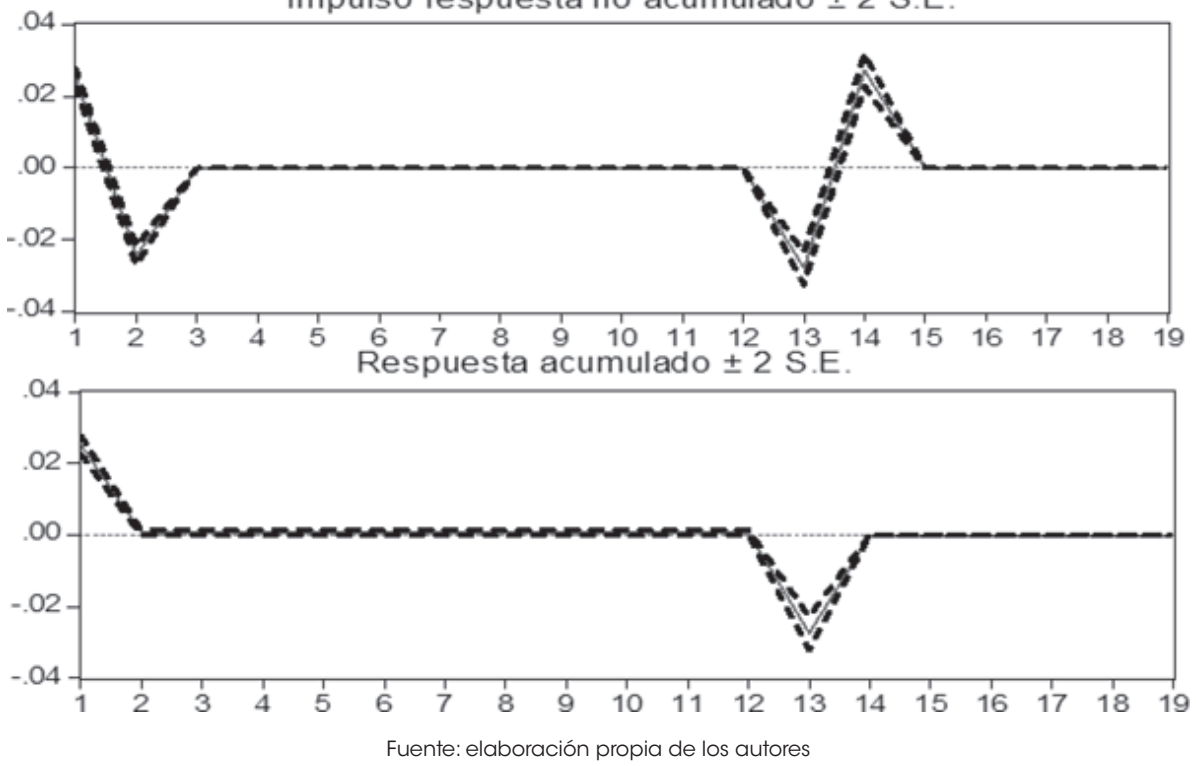

En el Gráfico 4, de las funciones impulso-respuesta para México y Brasil, podemos advertir que la respuesta acumulada y sin acumular de la tasa de depreciación debida al impulso de un shock equivalente al valor de una unidad de desviación estándar de la innovación, es positiva, estadísticamente significativo y además desaparece en forma asintótica en un lapso no más de 3 meses en promedio para México y 5 meses para Brasil.

Sin embargo, hay que recordar que el estadístico para la prueba de efectos $\mathrm{ARCH}$, que evalúa si la varianza de los residuos $\varepsilon_{\mathrm{t}}$ del modelo SARIMA de los cuadros 1 y 2 , es constante. La prueba indica que se rechace la hipótesis nula, en vista de lo cual se puede modelar el proceso de varianza condicional para los residuos por medio de modelos autorregresivos con heterocedasticidad condicional (ARCH), y así tendríamos una estimación de la volatilidad cambiaria. 


\subsection{Resultados de estimación de la volatilidad cambiaria}

\section{Cuadro 4 \\ Modelos estimados para la ecuación de la varianza}

\begin{tabular}{|c|c|}
\hline Modelo & Ecuación \\
\hline $\begin{array}{l}\text { Modelo (1) } \\
\text { Arch (1) } \\
\text { México }\end{array}$ & $\begin{aligned} \sigma_{t}^{2}= & 0.00054+0.12 \varepsilon_{t-1}^{2} \\
& (9.34)\end{aligned}$ \\
\hline $\begin{array}{l}\text { Modelo (2) } \\
\text { Arch (1) } \\
\text { Brasil }\end{array}$ & $\begin{array}{c}\sigma_{t}^{2}=0.00087+0.35 \varepsilon_{t-1}^{2} \\
(7.78)\end{array}$ \\
\hline $\begin{array}{l}\text { Modelo (3) } \\
\text { Garch }(1,1) \\
\text { México }\end{array}$ & $\begin{aligned} \sigma_{t}^{2}= & 0.00016+0.09 \varepsilon_{t-1}^{2}+0.64 \sigma_{t-1}^{2} \\
& (1.73)\end{aligned}$ \\
\hline $\begin{array}{l}\text { Modelo (4) } \\
\text { Garch }(1,1) \\
\text { Brasil }\end{array}$ & $\begin{aligned} \sigma_{t}^{2}= & 0.00068+0.31 \varepsilon_{t-1}^{2}+0.18 \sigma_{t-1}^{2} \\
& (2.48)\end{aligned}$ \\
\hline $\begin{array}{l}\text { Modelo (5) } \\
\text { Tgarch }(1,1) \\
\text { México }\end{array}$ & $\begin{aligned} \sigma_{t}^{2}= & 0.00012+0.16 \varepsilon_{t-1}^{2}+0.31 \varepsilon_{t-1}^{2} d_{t-1}+0.81 \sigma_{t-1}^{2} \\
& (3.74)\end{aligned}$ \\
\hline $\begin{array}{l}\text { Modelo (6) } \\
\text { Tgarch }(1,1) \\
\text { Brasil }\end{array}$ & $\begin{aligned} \sigma_{t}^{2}= & 0.00071-0.23 \varepsilon_{t-1}^{2}+0.19 \varepsilon_{t-1}^{2} d_{t-1}+0.16 \sigma_{t-1}^{2} \\
& (2.49)\end{aligned}$ \\
\hline $\begin{array}{l}\text { Modelo (7) } \\
\text { EGarch } \\
\text { México }\end{array}$ & $\begin{array}{r}\ln \sigma_{t}^{2}=-1.67+0.77 \ln \sigma_{t-1}^{2}+0.35 \frac{\varepsilon_{\mathrm{t}-1}}{\sigma_{\mathrm{t}-1}^{2}}-0.25 \frac{\left|\varepsilon_{\mathrm{t}-1}\right|}{\left|\sigma_{\mathrm{t}-1}^{2}\right|} \\
(-2.79)\end{array}$ \\
\hline $\begin{array}{l}\text { Modelo (8) } \\
\text { EGarch } \\
\text { Brasil }\end{array}$ & $\begin{aligned} \ln \sigma_{t}^{2}= & -12.01-0.73 \ln \sigma_{t-1}^{2}-0.20 \frac{\varepsilon_{\mathrm{t}-1}}{\sigma_{\mathrm{t}-1}^{2}}+0.79 \frac{\mid \varepsilon_{\mathrm{t}-1}}{\mid \sigma_{\mathrm{t}-1}^{2}} \\
& (-126.86) \quad(-18.40)\end{aligned}$ \\
\hline
\end{tabular}

$4 \quad$ La variable explicada en todos los modelos del Cuadro 2 se refiere a la volatilidad condicional del tipo de cambio estimado con los distintos modelos de las familias ARCH. 


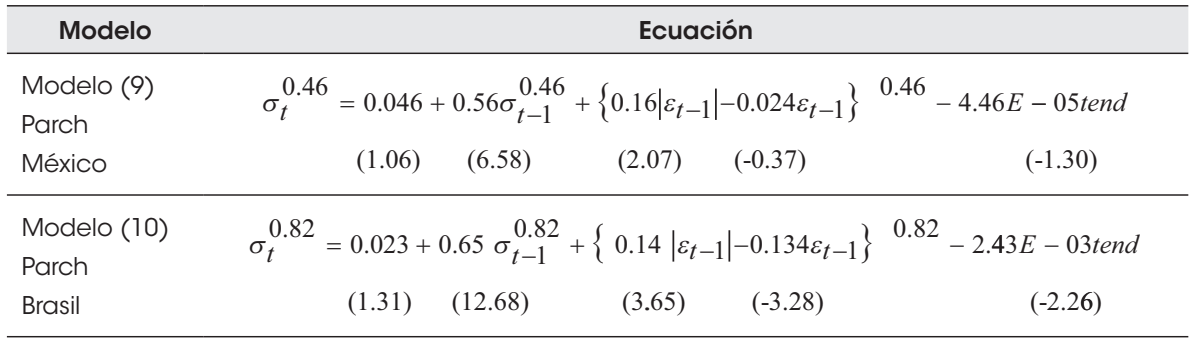

Fuente: elaboración propia de los autores, ( ) estadístico Z

Los modelos han sido estimados para el período muestral mensual que abarca desde enero de 1996 hasta diciembre de 2013, para México, y desde enero de 1994 hasta agosto de 2014, para Brasil; en ambos casos se manejan más de 200 observaciones. La estimación se ha realizado utilizando el método de la máxima verosimilitud, suponiendo que una distribución de errores generalizados, de acuerdo con la propuesta de Nelson (1991) teniendo en cuenta que no puede admitirse que los datos considerados estén normalmente distribuidos.

La estimación de los modelos (1) para México y (2) para Brasil, del Cuadro 3, por medio del método del quasimáximo de verosimilitud, proporciona los resultados presentados en el Cuadro 3. Se puede ver que los parámetros estimados $\beta_{Q M V}$ son significativos, ya que tienen un Z-estadístico mayor a dos en valor absoluto. También en el mismo modelo ARCH (1) se puede advertir que se cumple el supuesto de estacionariedad débil del proceso para ambos países: $\sum_{i=1}^{t} \varepsilon_{i}^{2}=0.12<1$ y $\sum_{i=1}^{t} \varepsilon_{i}^{2}=0.35<1$. Al mismo tiempo, los resultados nos señalan que el mejor moldelo que se ajusto a la información mensual fue un modelo ARCH (1), ya que éste presentó en valor absoluto los valores más grandes de criterios estadísticos ${ }^{5}$ no paramétricos, como el criterio de información Akaike (AIC) y el de Schwarz (SCH) con relación a otros procesos ARCH de distinto orden.

Por otro lado, los resultados enmarcados en el Cuadro 3 (modelos 3 y 4 ) muestran que el proceso GARCH $(1,1)$ fue significativo para la mayoría de los parámetros de ambos países, utilizando un nivel de significancia del Z-estadístico mayor a dos en valor absoluto. De igual manera, podemos apreciar que se cumple la condición de estacionariedad débil del proceso GARCH $(1,1)-M\left(\sum \alpha_{1}+\gamma_{1}<1\right)$, y la misma sugiere que la volatilidad cambiaria, tanto en México como en Brasil, tiende a disminuir con el paso del tiempo, que la misma puede

5 Con criterios estadísticos no paramétricos nos referimos a Schwarzcriterion, Akaikeinfocriterion, etc., que son criterios que nos permiten seleccionar entre dos o más modelos que compiten. En el caso de los modelos ARCH, se estimaron modelos ARCH (1), ARCH (2), ARCH (3) y ARCH (4), y el mejor resultó ser un modelo ARCH (1). 
explicarse por la utilización de la intervención esterilizada en los mercados cambiarios que utiliza con frecuencia el Banco de México para mitigar fundamentalmente depreciaciones no deseadas (Mántey, 2009: 72), y así lograr oportunamente el cumplimiento de la meta de inflación deseada.

Asimismo, los resultados enmarcados en el Cuadro 3 (modelos 5 y 6 ) muestran que el proceso TGARCH $(1,1)$ fue significativo en la mayoría de los parámetros de ambos países, utilizando un nivel de significancia del Z-estadístico mayor a dos en valor absoluto.

Además, se puede argumentar que, tanto para México como para Brasil, el componente Threshold es $\left(\varepsilon_{t-1}^{2} x d_{t-1}\right)>0$; ello implica que existe efecto leverage y/o apalancamiento, y si este Threshold ( $\left.\varepsilon_{t-1}^{2} x d_{t-1}\right)$ es distinto de cero, sugeriría la existencia de efectos asimétricos de la depreciación cambiaria sobre la volatilidad del tipo de cambio. Es decir que la volatilidad cambiaria en México y Brasil ${ }^{6}$ ha tendido a incrementarse más cuando la depreciación cambiaria supera las expectativas que cuando ésta es menor a la esperada. Ahora bien, con relación al componente $\varepsilon_{t-1}^{2}$, resulta significativo para México y no así para Brasil, y su signo nos confirmaría, en el caso de México, que los shocks positivos tienen un efecto positivo sobre la varianza condicional del tipo de cambio. Mientras que en el caso de Brasil nos afirmaría que los shocks positivos tienen un efecto adverso sobre la varianza condicional del tipo de cambio, y si sumamos los componentes $\left(\varepsilon_{t-1}^{2} ; \varepsilon_{t-1}^{2} x d_{t-1}\right)$, tendremos el efecto de los shocks negativos.

Asimismo en el Cuadro 3 (modelos 7 y 8) se muestra que el proceso EGARCH fue significativo para el periodo de análisis en la mayoría de los casos para ambos países, utilizando un nivel de significancia del Z-estadístico mayor a dos en valor absoluto. Ahora bien, con relación al componente $\varepsilon_{\mathrm{it}-1} / \sigma_{\mathrm{it}-1}^{2}$, como es distinto de cero en ambos modelos (3 y 4) tanto para México como para Brasil, nos afirma que, primero, los shocks son asimétricos, luego, como es significativo y con signo positivo para México, implica que shocks positivos de depreciación cambiaria tienen un impacto mayor sobre la volatilidad cambiaria que shocks negativos de la misma magnitud. De hecho, para el caso de México, el efecto estimado de los shocks positivos en el presente modelo EGARCH tiene el mismo signo y los shocks son significativos al estimado en el modelo TGARCH, 0.35 vs 0.16 , respectivamente. En esa línea, como es significativo y con signo negativo para Brasil, implica que shocks positivos de depreciación cambiaria tienen un impacto menor sobre la volatilidad cambiaria que shocks negativos de la misma magnitud; y, también para el caso de Brasil, el efecto estimado de los

6 En el caso de México, el componente Threshold es significativo, ya que tiene un estadístico Z mayor a dos en valor absoluto; no así en Brasil. 
shocks positivos en el presente modelo EGARCH tiene el mismo signo y los shocks son significativos al estimado en el modelo TGARCH, -0.20 vs -0.23 .

Los resultados de los modelos TGARCH y EGARCH (modelos 5, 6, 7 y 8) para México y Brasil podrían explicarse por el hecho que en México se trata de mantener al tipo de cambio en una senda de apreciación, para el cumplimiento de la meta de inflación deseada, mediante la utilización de la intervención esterilizada en los mercados cambiarios Mántey (2006 y 2009). Mientras que para Brasil, se utiliza la política monetaria y cambiaria para evitar mayores apreciaciones no deseadas que vayan a ir en desmedro de sus exportaciones y su balanza comercial.

Cuadro 5

Estadísticos de diagnóstico de los modelos estimados

\begin{tabular}{lllllll}
\hline \multicolumn{1}{c}{ Modelo } & Akaike & Schwarz & Hannan-Quinn & $\mathbf{R}^{2}$ & Arch-Pvalue & Theil \\
\hline México & & & & & & \\
\hline ARCH (1) & -4.51 & -4.40 & -4.23 & 0.77 & 0.94 & 0.017 \\
\hline GARCH & -4.51 & -4.39 & -4.18 & 0.76 & 0.96 & 0.018 \\
\hline TGARCH & -4.60 & -4.46 & -4.64 & 0.73 & 0.69 & 0.018 \\
\hline EGARCH & -4.60 & -4.46 & -4.39 & 0.77 & 0.52 & 0.020 \\
\hline PARCH & -4.57 & -4.41 & -4.40 & 0.71 & 0.23 & 0.017 \\
\hline Brasil & & & & & & 0.94 \\
\hline ARCH (1) & -3.82 & -3.68 & -2.58 & 0.67 & 0.017 \\
\hline GARCH & -3.93 & -3.75 & -2.67 & 0.69 & 0.96 & 0.018 \\
\hline TGARCH & -3.80 & -3.64 & -2.64 & 0.65 & 0.69 & 0.018 \\
\hline EGARCH & -3.91 & -3.71 & -2.39 & 0.66 & 0.52 & 0.020 \\
\hline PARCH & -3.79 & -3.60 & -2.40 & 0.67 & 0.23 & 0.017 \\
\hline
\end{tabular}

Fuente: elaboración propia de los autores

Tal y como se observa, según los criterios estadísticos no paramétricos Akaike y Schwarz, los modelos con mejor ajuste en la muestra del Cuadro 5 son el modelo asimétrico EGARCH, para México, y el modelo simétrico GARCH, para Brasil. La diferencia entre estos dos modelos reside en que el GARCH impone simetría en los shocks de depreciación cambiaria. Es decir que el coeficiente del término ARCH $(\alpha 1)$ del Modelo GARCH $(1,1)$ de la ecuación 4 , recoge los efectos de los shocks sin distinguir entre el signo de los mismos. Si la varianza condicional de los errores responde de manera distinta a shocks negativos y positivos, se estaría incurriendo en un error de especificación en la especificación $\operatorname{GARCH}(1,1)$. 
Por su parte, como se mencionó, los modelos asimétricos como el EGARCH permiten acomodar la asimetría producida por el "efecto apalancamiento" al capturar el efecto más fuerte que tienen los rendimientos negativos en la volatilidad. Por ejemplo, el modelo TGARCH o ARCH por umbrales de Zakoian (1990) y Glosten, Jagannathan y Runkle (1993).

Gráfico 5: Volatilidad estocástica del tipo de cambio (izquierda) y tasa de depreciación nominal del tipo de cambio peso-dólar en México (derecha)

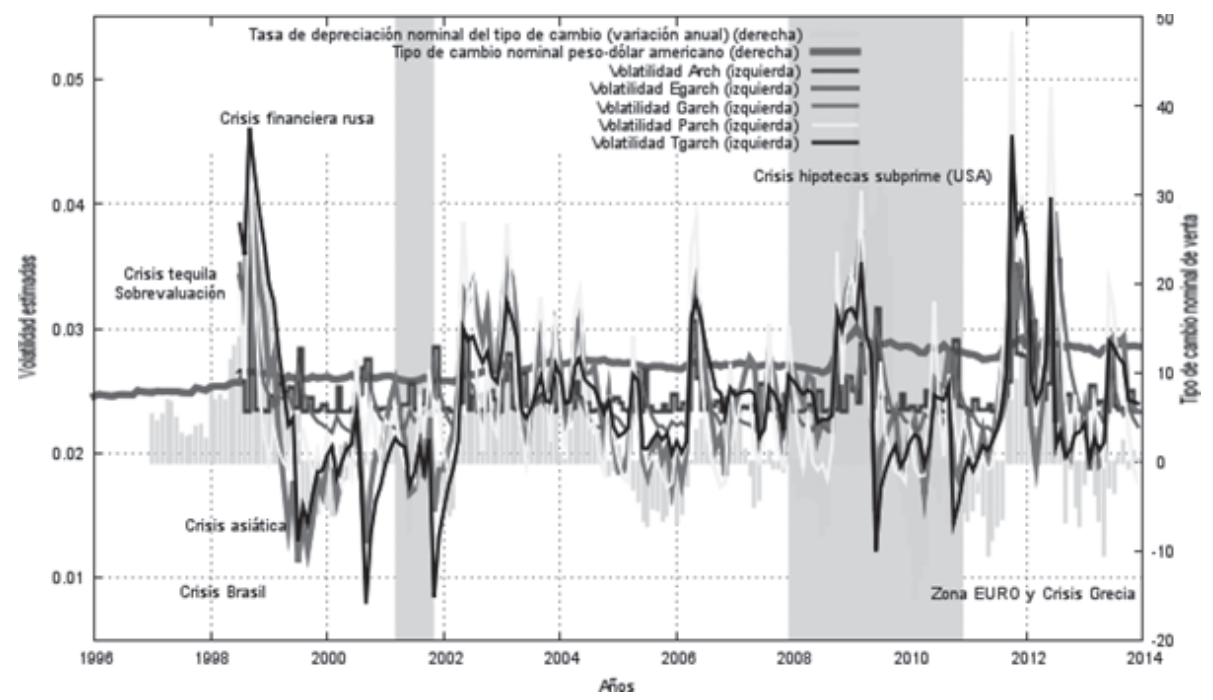

Fuente: elaboración propia de los autores 
Gráfico 6: Volatilidad estocástica del tipo de cambio (izquierda) y tasa de depreciación nominal del tipo de cambio real-dólar en Brasil (derecha)

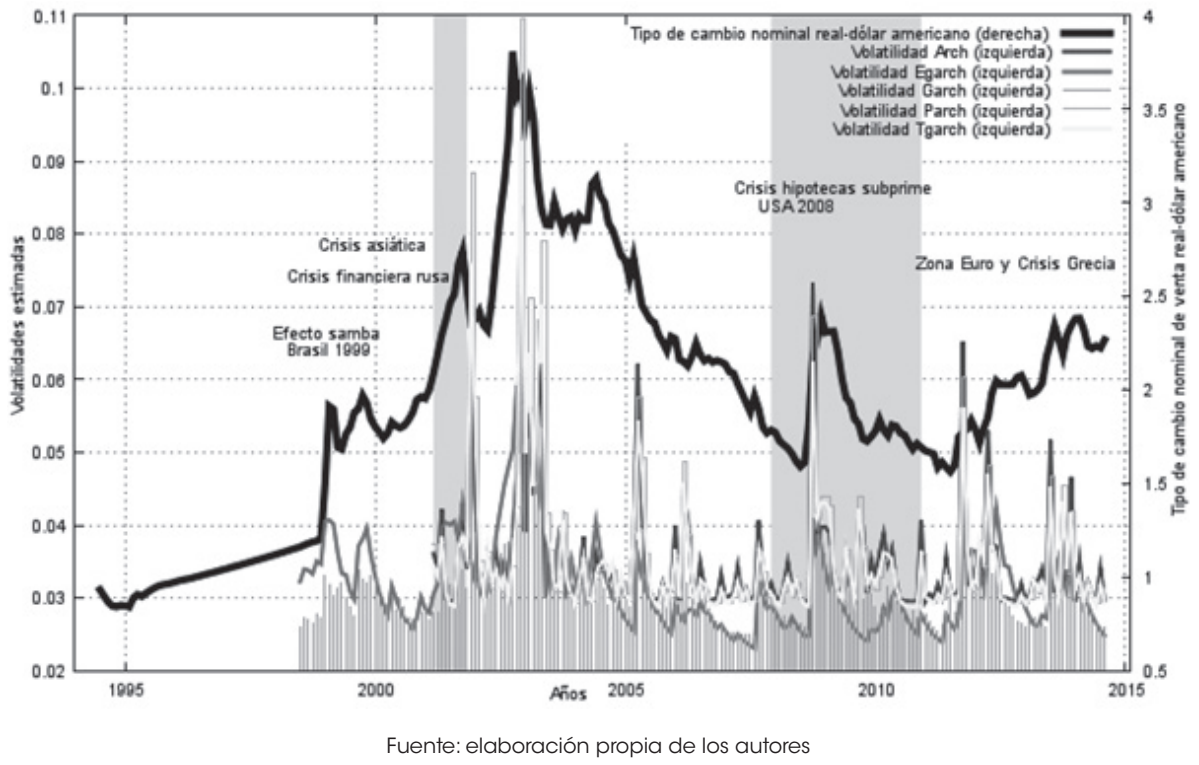

En los gráficos 5 y 6 se pueden advertir los distintos sucesos económicos y sociales que han provocado un alza y/o baja en la volatilidad cambiaria de México y Brasil. Así, por ejemplo, tenemos el caso de la "crisis del tequila", que, debido a los hechos políticos, sociales y económicos asociados a 1994, dieron lugar a una triple crisis, financiera, cambiaria y bancaria, a fines de ese año. Un común denominador para esa crisis es que se debió fundamentalmente al mantenimiento de un ancla cambiaria que sobrevaluó en demasía la moneda Mántey (2009). Esto provocó la devaluación del peso mexicano en diciembre de 1994, a la se aunaron los bajos niveles de reservas internacionales y la creciente movilidad de los flujos internacionales de capital. Por ello, las autoridades monetarias de México vieron conveniente trabajar sobre la base de un régimen de flotación libre y el origen del marco de política monetaria de metas explícitas de inflación?

Asimismo, tenemos otros periodos de volatilidad en la última década del siglo XX, por ejemplo, en los años 1997, 1998 y 1999, los mismos que pueden atribuirse a la crisis de los "tigres asiáticos", ocurrida a mediados de 1997, la crisis de la deuda rusa, en 1998, y el "efecto

7 A partir de 1993 se quitaron tres ceros a la moneda mexicana y se pasó a denominarla nuevo peso (Banxico, 1993). 
samba" (crisis del real brasileño), en 1999. Por otro lado, los periodos de alta volatilidad de principios del siglo XXI (años 2000 y 2001), pueden deberse en gran medida a la desaceleración de la economía norteamericana (recesión) a través de la denominada crisis de las empresas "punto com" y el efecto adverso que tuvo la misma sobre variables reales, generando incertidumbre. También, se puede advertir que los años 2005, 2006 y 2007 son periodos de volatilidad controlada.

A mediados del 2008, 2009 y 2010 se observan nuevamente agrupamientos de una elevada volatilidad cambiaria, la génesis de la cual es la crisis de las hipotecas subprime, que se inició en Estados Unidos y que contagio a una buena parte de países a nivel mundial. A ello se sumó la crisis griega de 2010, provocando incertidumbre en el ritmo de depreciación cambiaria y una elevada volatilidad. Por el lado del mercado financiero mexicano, los flujos de capital se contrajeron de forma significativa, lo que dio lugar a una alta volatilidad del tipo de cambio ${ }^{8}$. Teniendo en consideración los riesgos que tiene para la estabilidad del sistema financiero un mercado cambiario altamente volátil, la Comisión de Cambios en México llevó a cabo diversas acciones para proveer de liquidez al mercado cambiario, y de esta manera asegurar su buen funcionamiento. Por último, la agudización de los problemas financieros en la Zona Euro y el deterioro de las expectativas de crecimiento en la economía mundial generaron, de nueva cuenta, presiones sobre el tipo de cambio en los últimos meses de 2011 y el segundo trimestre de 2012; por lo mismo, la moneda mexicana se ubicó por encima de los 14.0 pesos por dólar.

Por su parte, el Gráfico 6 permite advertir los distintos sucesos económicos y sociales que han provocado un alza y/o baja en la volatilidad cambiaría de Brasil. Así, por ejemplo, tenemos los años 1997, 1998 y 1999, correspondientes a la crisis de los "tigres asiáticos", ocurrida a mediados de 1997, la crisis de la deuda rusa, en 1998, y el "efecto samba" (crisis del real brasileño), en 1999, y se puede advertir cómo estas tres crisis están interrelacionadas entre sí, hecho explicado en parte por el flujo comercial que tiene Brasil con esos países. Por otro lado, los periodos de alta volatilidad de principios del siglo XXI (años 2001, 2002 y 2003) pueden deberse en gran medida, a la desaceleración de la economía norteamericana (recesión) a través de la denominada crisis de las empresas "punto com" y el efecto adverso que tuvo la misma sobre variables reales, generando incertidumbre.

8 Para septiembre de 2008, el peso mexicano muestra alta volatilidad frente al dólar americano, cotizándose en 10.9814 pesos por dólar, lo que representa una devaluación de $6.77 \%$ respecto al mes anterior. En diciembre se incrementa a 14.3097 pesos, hasta alcanzar 15.365 pesos por dólar el 3 de marzo de 2009, con una devaluación de $40.52 \%$ con respecto al mismo mes del año anterior (Banxico, 2008). 
A mediados de 2008, 2009 y 2010 se observan nuevamente agrupamientos de una elevada volatilidad cambiaria, la génesis de la cual es la crisis de las hipotecas subprime, que se inició en Estados Unidos y que contagió a una buena parte de países a nivel mundial. A ello se sumó la crisis griega de 2010.

\subsection{Aplicación empírica al caso mexicano y brasileño (enfoque Switching Regression)}

\subsubsection{Modelos con probabilidad de transición variable}

Si nuestro objetivo es analizar, estimar y pronosticar la volatilidad cambiaria, los modelos de las familias GARCH son una buena alternativa a adoptar. En cambio, si se busca contar con información respecto a las probabilidades de observar baja o alta volatilidad cambiaria, la persistencia de las mismas, la probable duración de cada uno de los estados, es decir, cuánto se espera que dure un periodo de alta (baja) volatilidad o cuál es la probabilidad de que se pase a un estado de alta volatilidad cuando el tipo de cambio se encuentra en el estado de baja volatilidad o viceversa, son en realidad los modelos estocásticos de cambio de régimen (Switching Regression Model) los que permiten responder a estos cuestionamientos.

En esa línea, el objetivo de esta sección es identificar los períodos de alta y/o baja probabilidad de la volatilidad cambiaria en México en el periodo 1996m01-2013m12 y Brasil 1994m07-2014m08, con datos mensuales, además de observar la influencia de variables de economía política en el cambio de regímenes cambiarios.

La variable dependiente $\left(\mathrm{Vc}_{\mathrm{t}}\right)$ en el modelo es la volatilidad del tipo de cambio nominal peso-dólar norteamericano (Modelo EGARCH) para México y real-dólar (Modelo GARH) para Brasil ${ }^{1}$. Por lo tanto, para determinar la selección óptima del rezago, se aplicó una estrategia que va de lo general a lo particular, empezando desde una especificación de $r=6$ retardos y detectando, dados los resultados de los cuadros 5 y 6 , que el modelo con el menor criterio de Akaike es un modelo MSAR (5) - e(1) para México y MSAR (4) - e(1) Brasil.

9 Debido a los mejores resultados obtenidos en los cuadros 3 y 4, respectivamente. 


\section{Cuadro 6}

Criterios de selección de retardos de estadísticos no paramétricos para el modelo MSAR (q) (México)

\begin{tabular}{ccccc}
\hline Rezago q & Akaike & Schwarz & Hannan-Quinn & Akaike/T \\
\hline 5 & -6.557 & -6.334 & -6.568 & -0.03036 \\
\hline 4 & -6.434 & -6.225 & -6.350 & -0.02979 \\
\hline 3 & -6.485 & -6.103 & -6.212 & -0.03002 \\
\hline 2 & -6.721 & -6.925 & -6.824 & -0.03112 \\
\hline 1 & -6.238 & -6.771 & -6.871 & -0.02888 \\
\hline
\end{tabular}

Fuente: elaboración propia de los autores

T: número de observaciones

\section{Cuadro 7}

Criterios de selección de retardos de estadísticos no paramétricos para el modelo MSAR (q) (Brasil)

\begin{tabular}{ccccc}
\hline Rezago q & Akaike & Schwarz & Hannan-Quinn & Akaike/T \\
\hline 5 & -8.357 & -8.134 & -8.368 & -0.04353 \\
\hline 4 & -8.234 & -8.425 & -8.350 & -0.04288 \\
\hline 3 & -8.821 & -8.225 & -8.424 & -0.04594 \\
\hline 2 & -8.844 & -8.522 & -8.823 & -0.04606 \\
\hline 1 & -8.338 & -8.171 & -8.571 & -0.04343 \\
\hline
\end{tabular}

Fuente: elaboración propia de los autores

T: número de observaciones

En la estimación con máxima verosimilitud de este modelo del Cuadro 7 se puede advertir que, mediante un proceso de transformación, el sigma del régimen 1 (baja volatilidad) es -8.23 para México y -6.77 para Brasil, mientras que el sigma del régimen 0 (alta volatilidad) es -5.63 para México y -5.38 para Brasil. Por lo tanto, el tipo de cambio nominal de venta tanto en México como en Brasil es más volátil en periodos de alta volatilidad que en periodos de baja volatilidad. También se puede advertir que las pruebas de efectos Arch y autocorrelación aplicados a los residuos del modelo MSAR (2) se aceptan, es decir que se cumplen los supuestos de no autorrelación y varianza constante de los residuos.

Al mismo tiempo, dentro de los factores comunes se puede advertir que solo el primer rezago de la variable explicada resulta significativo al $1 \%$. Asimismo, como dijimos en un principio, la varianza de los residuos que cambia entre estados de alta y baja volatilidad resulta significativa al 1\%, lo que es sinónimo de un cambio de régimen significativo y al mismo tiempo avalaría también la ejecución de un modelo con cambio de régimen (Markov Switching 
Regresión Model). En esa misma línea, la tasa de depreciación presenta signo positivo aunque no significativo solo en el régimen 0 de alta volatilidad. Esto nos confirmaría la relación directa y positiva que existe entre la volatilidad cambiaria y la tasa de depreciación. Por último, la dummy de tendencia y algunas dummys estacionales centradas resultan solo significativas en algunos periodos; en particular, el signo de la dummy de tendencia en ambos países nos diría que la volatilidad cambiaria habría disminuido durante el periodo en estudio y este resultado puede deberse en gran medida a la utilización de la intervención esterilizada en los mercados cambiarios, para mantener el tipo de cambio en la dirección que lo requiera la autoridad monetaria.

\section{Cuadro 8}

Estimaciones del modelo MSAR (2) y pruebas de diagnóstico del modelo para México Estimaciones del modelo MSAR (2) y pruebas de diagnóstico del modelo para Brasil

\begin{tabular}{|c|c|c|c|c|}
\hline \multirow{2}{*}{ Variable } & Coeficiente & Probabilidad & Coeficiente & Probabilidad \\
\hline & \multicolumn{2}{|c|}{ México } & \multicolumn{2}{|c|}{ Brasil } \\
\hline \multicolumn{5}{|c|}{ Régimen 0 (alta volatilidad) } \\
\hline C & 0.02123 & 0.0000 & 0.01260 & 0.0000 \\
\hline Tasa de depreciación & 0.00059 & 0.6312 & 0.00314 & 0.5046 \\
\hline LOG(SIGMA) & -5.62995 & 0.0000 & -5.38331 & 0.0000 \\
\hline \multicolumn{5}{|c|}{ Régimen 1 (baja volatilidad) } \\
\hline C & 0.01590 & 0.0000 & 0.00895 & 0.0000 \\
\hline Tasa de depreciación & -0.00025 & 0.9419 & -0.00087 & 0.7902 \\
\hline LOG(SIGMA) & -8.23343 & 0.0000 & -6.77292 & 0.0000 \\
\hline \multicolumn{5}{|c|}{ Factores comunes } \\
\hline Volatilidad (-1) & 0.45179 & 0.0000 & 0.72472 & 0.0000 \\
\hline Volatilidad (-2) & -0.12287 & 0.1281 & -0.02032 & 0.8081 \\
\hline Tendencia & $-6.386 e-007$ & 0.7962 & $-1.249 e-006$ & 0.7174 \\
\hline Destacional_centrada & -0.00066 & 0.2841 & -0.00109 & 0.2274 \\
\hline Destacional_centrada_1 & -0.00028 & 0.6502 & -0.00149 & 0.0567 \\
\hline Destacional_centrada_2 & -0.00093 & 0.2015 & -0.00058 & 0.0423 \\
\hline Destacional_centrada_3 & -0.00038 & 0.6852 & $-2.086 e-005$ & 0.9824 \\
\hline Destacional_centrada_4 & -0.00062 & 0.0000 & -0.00030 & 0.7425 \\
\hline Destacional_centrada_5 & 0.00958 & 0.0000 & -0.04107 & 0.0342 \\
\hline Destacional_centrada_6 & 0.00358 & 0.0151 & -0.02704 & 0.0482 \\
\hline
\end{tabular}




\begin{tabular}{|c|c|c|c|c|}
\hline \multirow{2}{*}{ Variable } & Coeficiente & Probabilidad & Coeficiente & Probabilidad \\
\hline & \multicolumn{2}{|c|}{ México } & \multicolumn{2}{|c|}{ Brasil } \\
\hline Destacional_centrada_7 & -0.00037 & 0.0002 & -0.00182 & 0.0503 \\
\hline Destacional_centrada_8 & 0.00053 & 0.0562 & -0.00064 & 0.4862 \\
\hline Destacional_centrada_9 & 0.00345 & 0.0234 & 0.00157 & 0.0603 \\
\hline Destacional_centrada_10 & -0.00242 & 0.0136 & -0.00106 & 0.2593 \\
\hline \multicolumn{5}{|c|}{ Matriz de parámetros de transición } \\
\hline POO (alta volatilidad) & 0.835935 & 0.0958 & 0.88141 & 0.1471 \\
\hline P11 (baja volatilidad) & 0.979993 & 0.0163 & 0.97713 & 0.0326 \\
\hline \multicolumn{2}{|l|}{ México } & \multicolumn{3}{|l|}{ Brasil } \\
\hline \multicolumn{2}{|c|}{ Logaritmo de verosimilitud: -223.2537 } & \multicolumn{3}{|c|}{ Logaritmo de verosimilitud: -423.6354} \\
\hline \multicolumn{2}{|c|}{ Número de observaciones: 185} & \multicolumn{3}{|c|}{ Número de observaciones: 192} \\
\hline \multicolumn{2}{|l|}{ Criterio de Akaike: -6.721} & \multicolumn{3}{|c|}{ Criterio de Akaike: - 8.844} \\
\hline \multicolumn{2}{|c|}{ Criterio de Hannan-Quinn: -6.824 } & \multicolumn{3}{|c|}{ Criterio de Hannan-Quinn: -8.823 } \\
\hline \multicolumn{2}{|l|}{ Criterio de Schwarz: -6.925} & \multicolumn{3}{|c|}{ Criterio de Schwarz: -8.522 } \\
\hline \multicolumn{2}{|c|}{$\begin{array}{l}\text { Prueba de Autocorrelación de Portmanteau } \\
\text { (p-value): } 0.9876\end{array}$} & \multicolumn{3}{|c|}{$\begin{array}{l}\text { Prueba de Autocorrelación de Portmanteau } \\
\text { (p-value): } 0.7538\end{array}$} \\
\hline \multicolumn{2}{|c|}{ Prueba Arch LM (p-value): 0.6164} & \multicolumn{3}{|c|}{ Prueba Arch LM (p-value): 0.3164} \\
\hline \multicolumn{2}{|c|}{ Media Volatilidad México: 0.0244} & \multicolumn{3}{|c|}{ Media Volatilidad Brasil: 0.03156} \\
\hline \multicolumn{2}{|c|}{ Desviación estándar Vol. México: 0.003138} & \multicolumn{3}{|c|}{ Desviación estándar Vol. Brasil: 0.005659} \\
\hline
\end{tabular}

Fuente: elaboración propia de los autores

Por otro lado, en el modelo MSAR (2) ${ }^{10}$ estimado para la volatilidad cambiaria de México y Brasil, podemos advertir que existe un buen ajuste entre el valor actual (gráfico superior para México y gráfico inferior para Brasil) y el valor pronosticado por nuestro modelo MSAR(2) (Gráfico 7). Este resultado implica un buen seguimiento del proceso generador de datos.

$\overline{10}$ Donde MSAR (2) es un modelo Markov Switching Autorregresivo de orden 2. 
Gráfico 7: Valor actual y pronosticado del modelo Switching para México y Brasil MSAR(2)
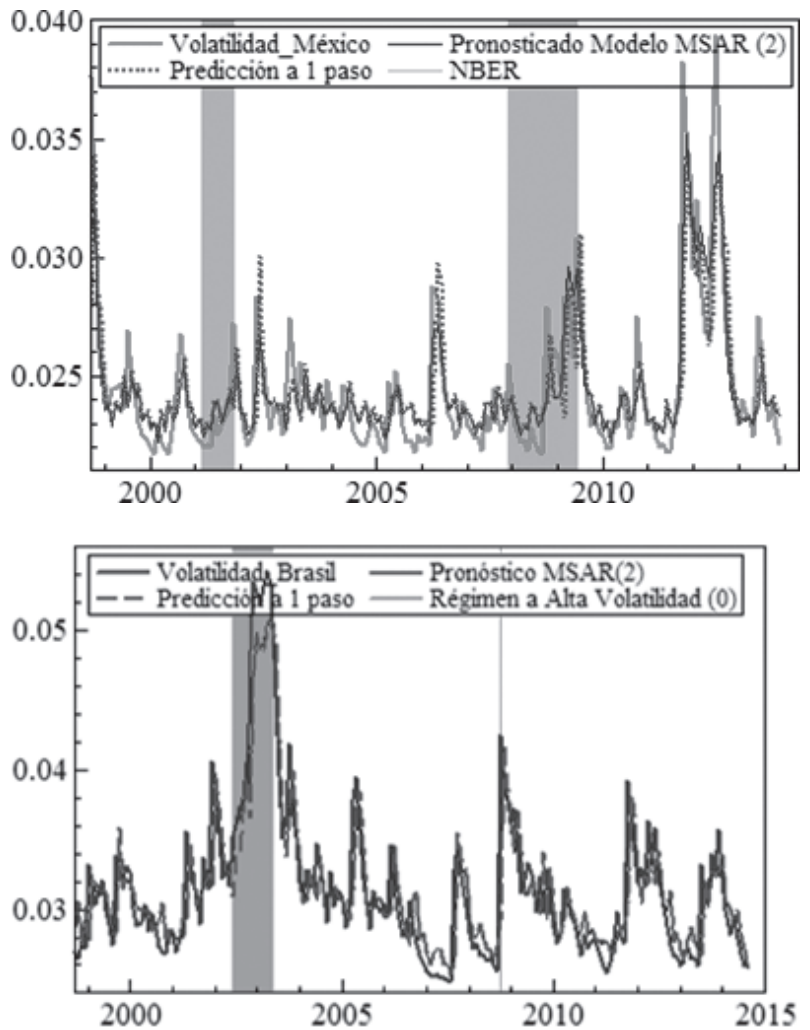

Fuente: elaboración propia de los autores

Cuadro 9

Matriz de probabilidades de transición

\begin{tabular}{ccccccc}
\hline \multicolumn{1}{c}{$t$} & \multicolumn{5}{c}{$t$} \\
\hline \multirow{4}{*}{$t-1$} & México & $\sigma$ baja & $\sigma$ alta & Brasil & $\sigma$ baja & $\sigma$ alta \\
\cline { 2 - 7 } & $\sigma$ baja & 0.97999 & 0.16407 & $\sigma$ baja & 0.97714 & 0.11859 \\
\cline { 2 - 7 } & $\sigma$ alta & 0.020007 & 0.83593 & $\sigma$ alta & 0.022865 & 0.88141 \\
\hline
\end{tabular}

Fuente: elaboración propia de los autores 


\section{Cuadro 10}

Duración promedio de cada estado (meses)

\begin{tabular}{rcccc}
\hline & \multicolumn{2}{c}{ México } & \multicolumn{2}{c}{ Brasil } \\
\hline Estado & $\begin{array}{c}\text { Duración } \\
\text { promedio }\end{array}$ & Desvío estándar & $\begin{array}{c}\text { Duración } \\
\text { promedio }\end{array}$ & Desvío estándar \\
\hline Estado $1^{11}$ & 50 & 49.47 & 44 & 43.24 \\
\hline Estado 0 & 6 & 5.57 & 8 & 7.92 \\
\hline
\end{tabular}

Fuente: elaboración propia de los autores

Lo que indican los cuadros 8 y 9 , respectivamente, es que los periodos de baja volatilidad en México y Brasil, en el periodo considerado, fueron relativamente persistentes con relación a los periodos de alta volatilidad. Por ejemplo, para México, la probabilidad de que una baja volatilidad esté seguida por otra de baja volatilidad en el siguiente mes es de 0.979 , por lo que estos periodos persistirían en promedio durante cincuenta meses (aproximadamente ocho semestres) y con una desviación estándar de 49.47. A su vez, para Brasil, la probabilidad de que una baja volatilidad esté seguida por otra de baja volatilidad en el siguiente mes es de 0.977, por lo que estos periodos persistirían en promedio durante cuarenta y cuatro meses (aproximadamente siete semestres) y con una desviación estándar de 43.24.

Por otro lado, para México, la probabilidad de que a una fase de alta volatilidad le siga otra de alta volatilidad en el siguiente mes es 0.836 , por lo que estos episodios persistirían unos seis meses ( 1 semestre) y con una desviación estándar de 5.57. A su vez, para Brasil, la probabilidad de que a una fase de alta volatilidad le siga otra de alta volatilidad en el siguiente mes es 0.882 , por lo que estos episodios persistirían unos ocho meses ( 1 semestre y 2 meses) y con una desviación estándar de 7.92.

Asimismo, para México, la probabilidad de pasar de un periodo de baja volatilidad a una de alta volatilidad es aproximadamente de 0.020 , y este resultado es menor que la probabilidad de pasar de un periodo de alta volatilidad a una de baja volatilidad (0.16). Por lo tanto, al observar la matriz de probabilidades de transición que se muestra en los cuadros 8 y 9 , se puede decir que una vez que la volatilidad del tipo de cambio peso-dólar se encuentra en un estado o régimen, es muy baja la probabilidad de que pase al otro estado. En particular, se observa que cuando se encuentra en el estado de baja volatilidad, es muy poco probable que pase al estado de alta volatilidad, siendo la probabilidad apenas superior a 2\%. Es relativamente mayor la probabilidad de que estando la volatilidad en el régimen de volatilidad alta pase al siguiente

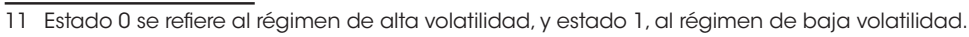


día al régimen de volatilidad baja (poco más de 16.5 por ciento). Para el caso brasileño, el análisis es similar, y se puede advertir que también es relativamente mayor la probabilidad de que, estando la volatilidad en el régimen de volatilidad alta, pase al siguiente día al régimen de volatilidad baja (aproximadamente 12\%).

Por último, se puede advertir que la persistencia estimada de la volatilidad cambiaria peso-dólar para México es del orden de 0.8159, y la del real-dólar, para Brasil, es de 0.8585. Ello implica una persistencia alta en volatilidad, y que los choques en ella no se disipan rápidamente, haciendo más riesgosa en términos del dólar la posición de un inversionista que se mantiene en moneda nacional. Este resultado explicaría en gran medida el proceso de extranjerización de ambas economías latinoamericanas, haciendo más atractivo y menos riesgoso invertir en dólares, así como la posibilidad de mantener los ahorros de las economías domésticas en moneda extranjera, todo ello debido al no abandono del ancla cambiaria hasta el día de hoy y al denominado "miedo a flotar" existente en ambas economías (Mántey, 2009).

Por otro lado, en el Gráfico 8 se muestran las funciones impulso-respuesta; en un modelo MSAR, correctamente especificado, se espera teóricamente que la respuesta ante una innovación desaparezca en forma asintótica, es decir, que tienda a cero en un horizonte de corto plazo; éste es un indicador de que el modelo es estacionario. Por su parte, el impulsorespuesta acumulado deberá tender al valor de largo plazo de la variable que se está modelando, también en un horizonte de corto plazo.

En este caso, podemos advertir que la respuesta sin acumular de la volatilidad cambiaria tanto en México como en Brasil, debido al impulso de un shock equivalente al valor de una unidad de desviación estándar de la innovación, es positivo, estadísticamente significativo y además desaparece en forma asintótica en un lapso de no más de 4 periodos en promedio para México y 10 periodos para Brasil ${ }^{12}$.

12 Este resultado confirmaría que el modelo estimado en este documento, MSAR (2), es estacionario y/o estable, tanto para México como para Brasil. 
Gráfico 8: Estabilidad del modelo Markov Switching MSAR (2) para México y Brasil (función impulso-respuesta sin acumular)
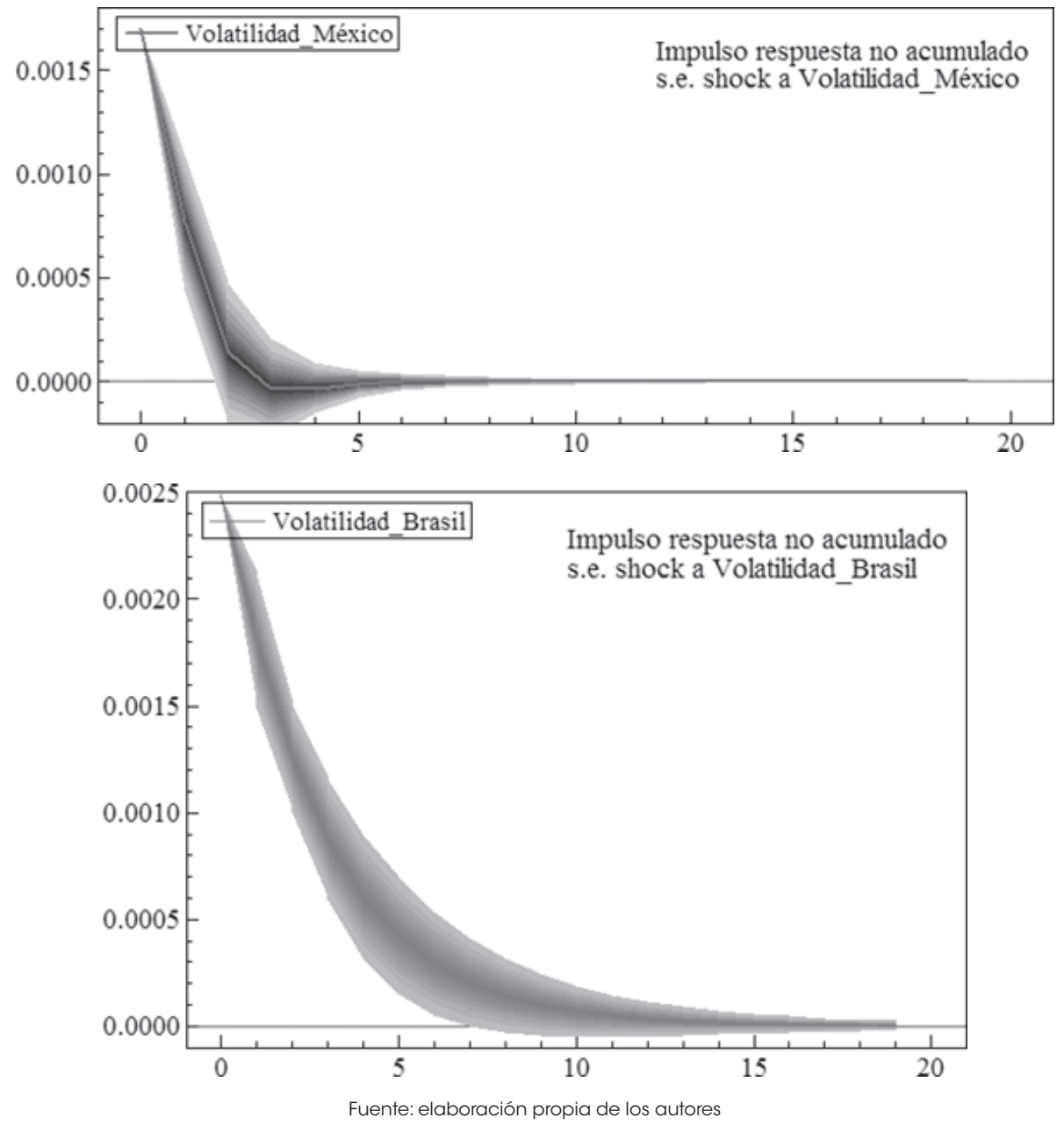
Gráfico 9: Probabilidad de estar en un estado de alta volatilidad (arriba izquierda), probabilidad de estar en un estado de baja volatilidad (arriba derecha) y estimación de Kernell (abajo) en México MSAR (2)

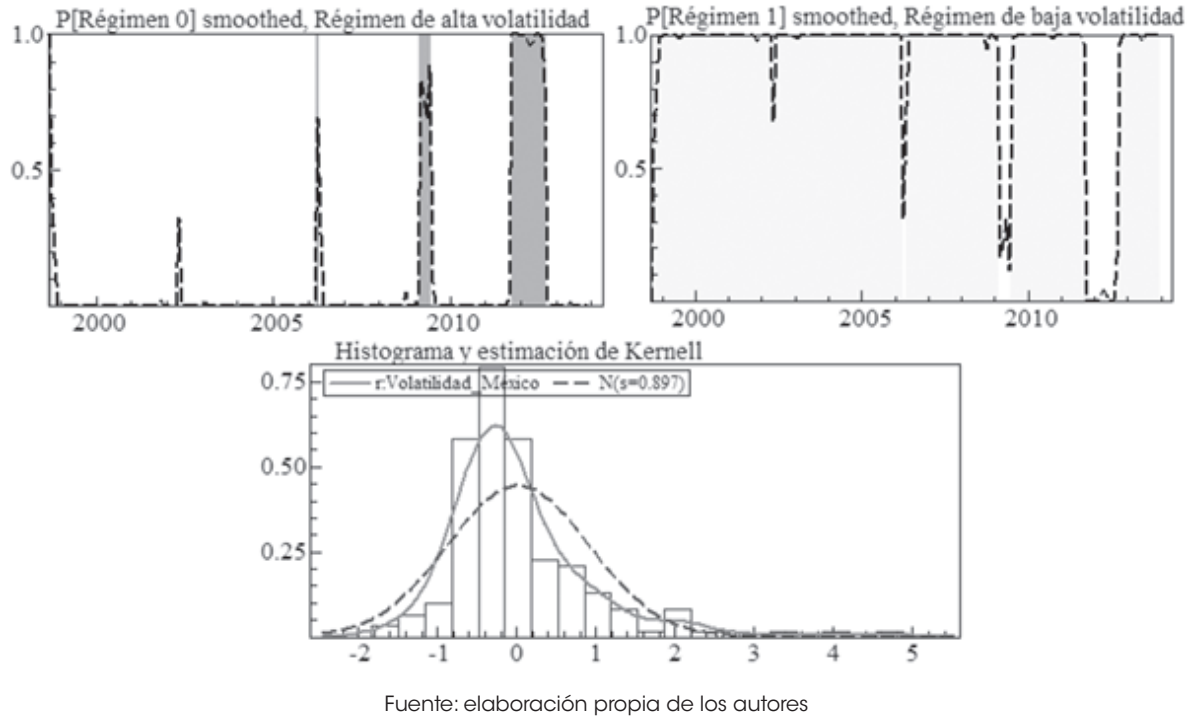

Gráfico 10: Identificación de regímenes de alta y baja volatilidad cambiaria en México, mediante la estimación de un modelo Markov Switching Autorregresivo de orden 2 MSAR(2)

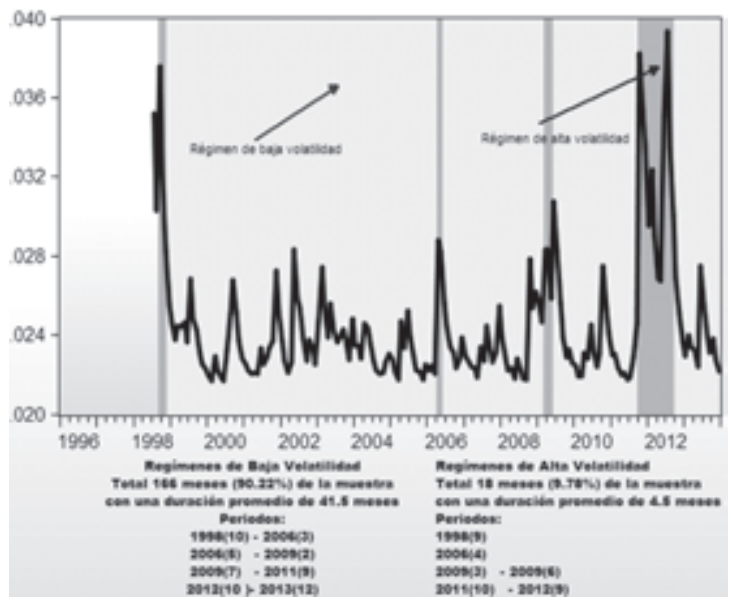

Fuente: elaboración propia de los autores 
En esta sección se ha analizado la volatilidad cambiaria peso-dólar para México y real dólar para Brasil, mediante la estimación de un modelo de volatilidad estocástica con un enfoque markoviano. Esta metodología permite identificar y caracterizar los periodos de baja y alta volatilidad mediante la estimación de las probabilidades de ocurrencia relacionados con cada uno de los estados (baja y alta volatilidad).

Asimismo, los gráficos 9 y 10 nos brindan la misma información con la metodología de los Switching Markov Regression Model. Por ejemplo, se observa que, según la estimación del modelo MSAR (2) para México, el año 1998 estuvo marcado por un alto nivel de volatilidad cambiaria, y por lo mismo la probabilidad de estar en un régimen de alta volatilidad es muy cercano a la unidad ${ }^{13}$; debido fundamentalmente a tres razones: en primer lugar, se atribuye responsabilidad al "efecto tequila" de 1994, pues a partir de este suceso económico, el peso sufrió importantes depreciaciones respecto del dólar ${ }^{14}$, debido a un ancla cambiaria que sobrevaluó en demasía el peso; en segundo lugar, se considera una causa la crisis asiática de julio de 1997 y la crisis financiera rusa de agosto de 1998, ambas con efectos en los mercados financieros mundiales, de los cuales México no está exento; por último, se considera el "efecto samba" (crisis brasileña) de principios de 1999, que de alguna u otra forma fue en desmedro de las economías latinoamericanas.

También para el caso mexicano se advierten pequeños saltos de volatilidad en los años 2000 y 2001, aunque el modelo MSAR(2) no los detecta, y por lo mismo no los considera significativos, ya que su probabilidad de estar en un régimen de alta volatilidad para este periodo es menor a 0.5. Pero estos saltos de volatilidad pueden deberse a la desaceleración de la economía de Estados Unidos (crisis de las empresas punto com). Dados los fuertes lazos comerciales que existen entre México y Estados Unidos, la crisis norteamericana del 2001 fue en detrimento de los intereses económicos mexicanos, con un efecto adverso sobre las exportaciones, la producción y el precio de la mezcla mexicana de petróleo.

También se observan alzas en la volatilidad cambiaria en los años 2006, 2009 y 2011(10)2012(9), cuyas probabilidades están en el orden del 0.75 para 2006, 0.80-0.85 para 2009 y prácticamente cercano a la unidad para el periodo 2011(10)-2012(9). La explicación que se puede dar a esos periodos es la crisis financiera global disparada por la crisis de las hipotecas subprime a inicios del 2007 y sus efectos en los mercados financieros mundiales,

13 Cabe recalcar que, según la teoría estadística, la probabilidad tiene un rango de variación de cero a uno.

14 En el caso de México, en octubre y noviembre de 1996 se observa un ascenso sostenido en el tipo de cambio, debido al cual se sitúa alrededor de ocho pesos por dólar. 
crisis desatada primero en Estados Unidos y extendida después a escala mundial. De manera general, se puede afirmar que, según la estimación del modelo MSAR(2) de los cuadros 7, 8 y 9, así como los modelos de las familias GARCH del Cuadro 3, en referencia al tipo de cambio peso-dólar para México, se observan periodos más o menos prolongados de baja volatilidad, interrumpidos por periodos cortos en los cuales la volatilidad es alta; esto se debería en parte a la intención del Banco de México de mantener el tipo de cambio en una senda de equilibrio (apreciación) mediante la utilización de varios instrumentos, entre ellos la intervención esterilizada en los mercados cambiarios.

En el caso de Brasil, se puede advertir que el modelo MSAR (2) detecta dos periodos significativos de alta volatilidad cambiaria, cuyas probabilidades son muy próximas a 1 para el periodo 2002-2003 y 0.8 para el año 2008. Estos periodos de alta volatilidad cambiaria vienen explicados en parte por la crisis económica de Estados Unidos de los años 2001 y 2002 y por la baja demanda de materias primas a nivel mundial en esos años. A su vez, la alta volatilidad cambiaria del año 2008 se debe a la crisis de las hipotecas subprime, que se origina en Estados Unidos y termina afectando a sus socios comerciales. A su vez, el Gráfico 12 nos señala que en gran parte de la muestra, para ser exactos, en un 93.23\% y con una duración promedio de 59.67 meses, predomina el régimen de baja volatilidad cambiaria, en parte explicado por la utilización de la intervención en los mercados cambiarios de parte del Banco Central de Brasil, en su intención de frenar la apreciación del real respecto del dólar y así no perjudicar a su balanza comercial y sus fuertes lazos comerciales con la República de China. 
Gráfico 11: Probabilidad de estar en un estado de alta volatilidad (arriba), probabilidad de estar en un estado de baja volatilidad (abajo izquierda) y estimación de Kernell (abajo derecha) en Brasil

$\operatorname{MSAR}(2)$
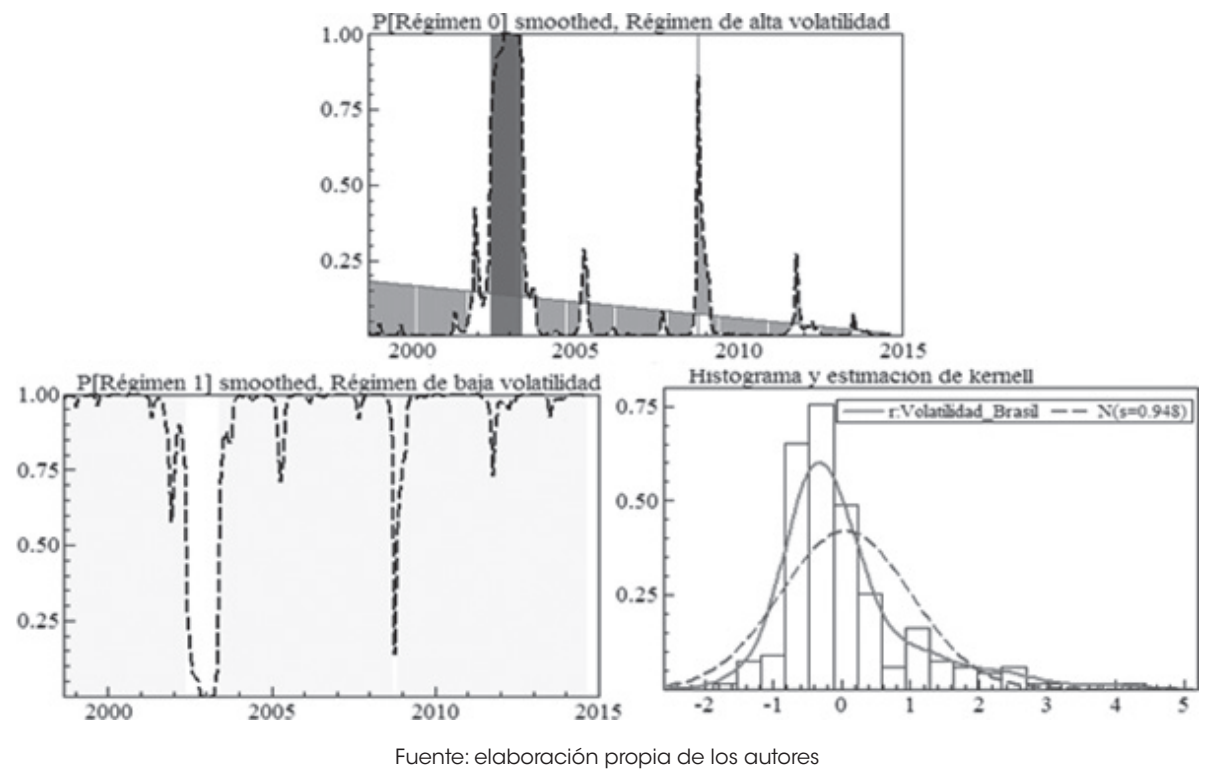

Gráfico 12: Identificación de regímenes de alta y baja volatilidad cambiaria en Brasil, mediante la estimación de un modelo Markov Switching Autorregresivo de orden 2 MSAR(2)

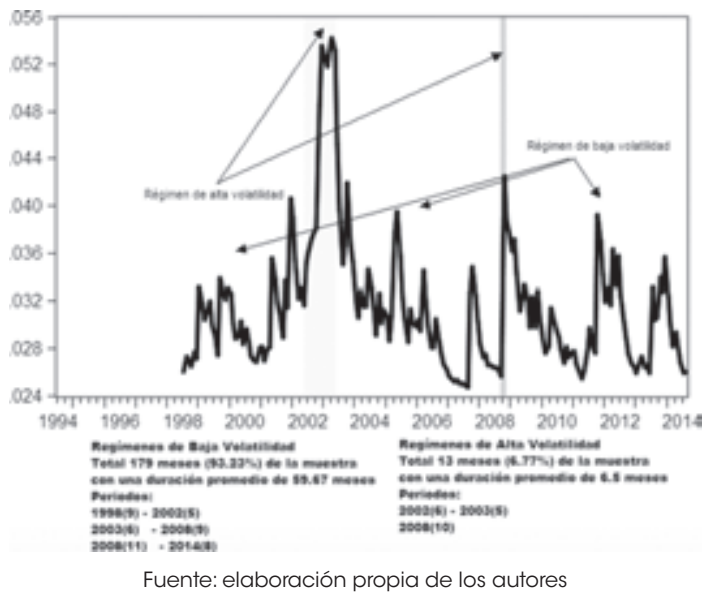


Por último, en los gráficos 13 y 14 se muestra el pronóstico dinámico de la volatilidad cambiaria y sus respectivos regímenes de alta y baja volatilidad en México y Brasil. En ambos países se puede advertir que la volatilidad fuera de la muestra de estudio tiende a aumentar levemente; esta situación se debería a la crisis internacional latente, en especial el de la zona euro y la crisis griega, que en distinto grado terminan afectando a distintas economías a nivel mundial.

\section{Gráfico 13: Pronóstico dinámico de la volatilidad y los regímenes cambiarios de alta y baja volatilidad, mediante la estimación de un modelo Markov Switching Autorregresivo de orden 2 MSAR (2) para México}
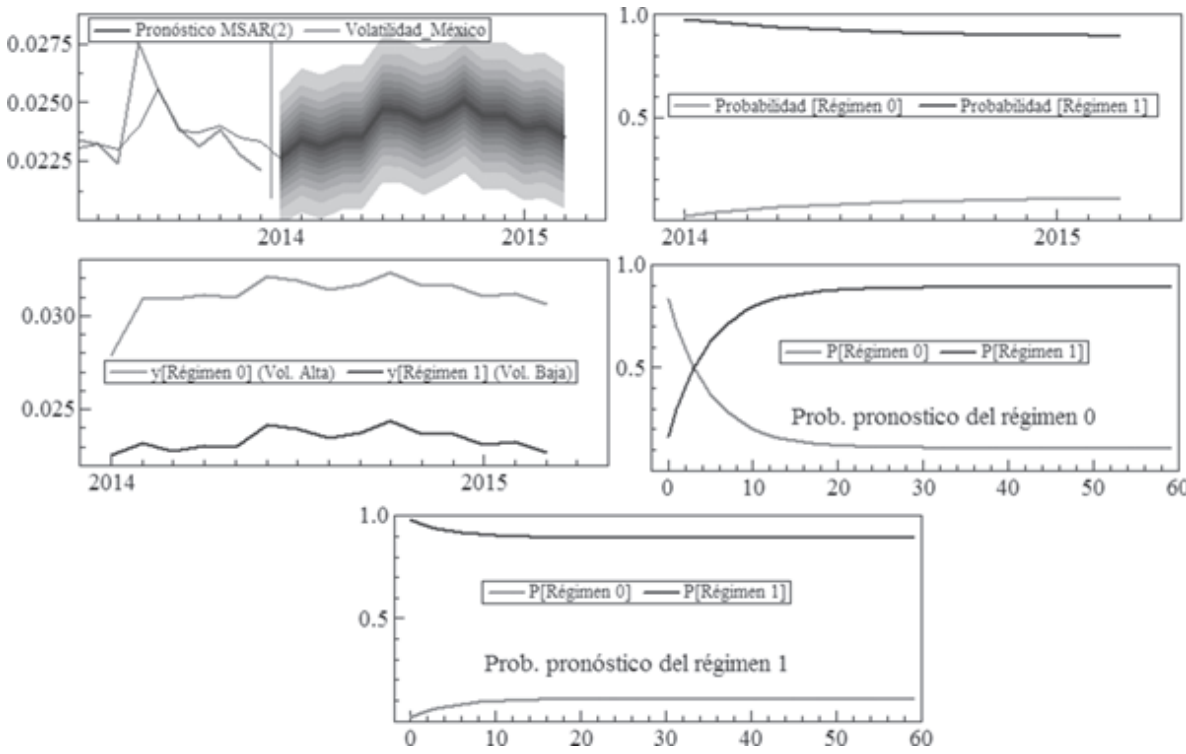

Fuente: elaboración propia de los autores 


\section{Gráfico 14: Pronóstico dinámico de la volatilidad y los regímenes cambiarios de alta y baja volatilidad, mediante la estimación de un modelo \\ Markov Switching Autorregresivo de orden 2 MSAR (2) para Brasil}
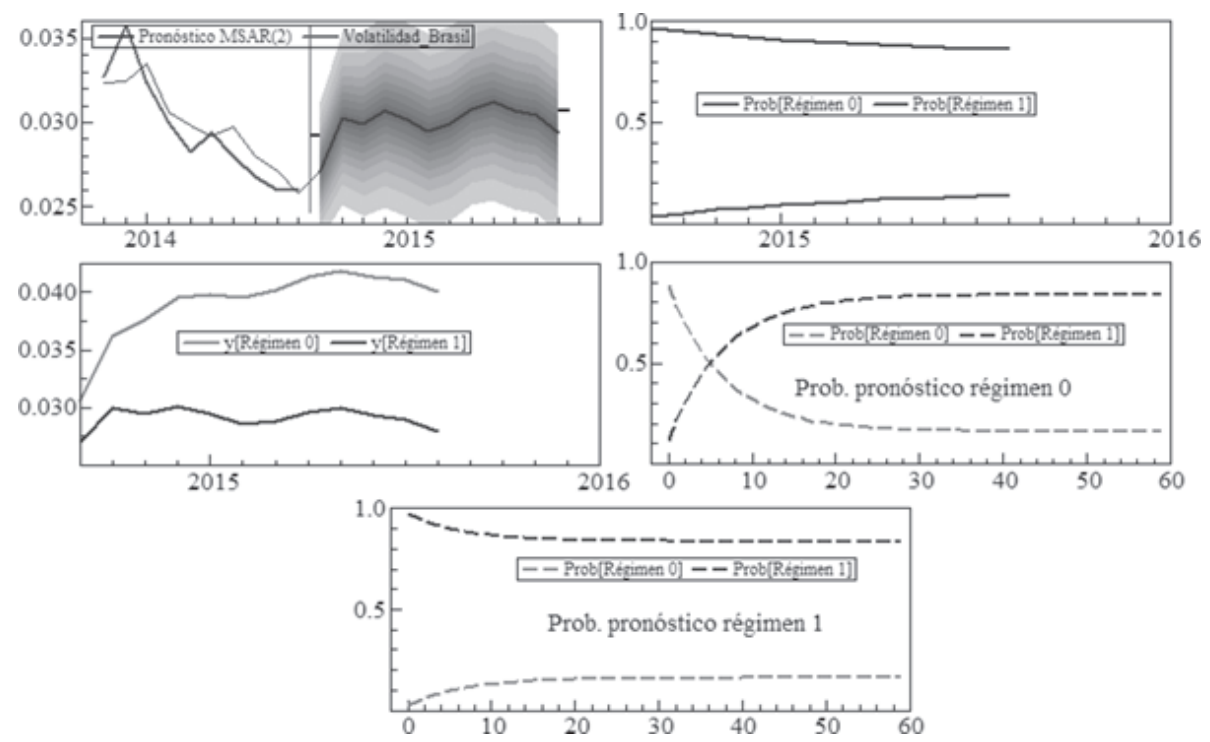

Fuente: elaboración propia de los autores

\section{Conclusiones}

El objetivo del presente documento ha sido analizar y estimar una medida adecuada de volatilidad cambiaria para México y Brasil, para lo cual inicialmente se estimaron modelos de volatilidad con las familias GARCH, ya que las mismas nos permiten analizar, estimar y pronosticar la volatilidad cambiaria. Sin embargo, en un entorno de volatilidad es imprescindible no solo conformarse con estimaciones de la volatilidad y su respectivo pronóstico, sino que es de gran preponderancia para los agentes económicos e inversores financieros contar con información respecto a las probabilidades que tienen de observar baja o alta volatilidad, la probable duración de cada uno de los estados y la persistencia de las mismas. Por esa razón se estimaron modelos Markov Switching Regression, ya que tienen esa ventaja con relación a los modelos de las familias GARCH. 
Asimismo, otro de los objetivos que ha perseguido el presente documento ha sido incluir las simetrías y asimetrías cambiarias a partir de distintos modelos de las familias GARCH, así como la utilización de los modelos Markov Switching Regression, ya que nos permite capturar el cambio de régimen de una determinada variable en estudio. Además, se ha elegido el dólar como referencia, por ser la moneda referente para la economía mexicana y brasileña. Los resultados, dentro del período muestral estudiado, indican que los modelos asimétricos analizados mejoran el ajuste realizado por el proceso $\operatorname{GARCH}(1,1)$ para el caso de México, mientras que para el caso de Brasil, es suficiente el modelo GARCH $(1,1)$ simétrico.

Otro resultado interesante de los modelos GARCH se debe a que la mayoría de los modelos de las familias GARCH sugiere que la volatilidad cambiaria tanto en México y Brasil tiende a disminuir con el paso del tiempo, y que la misma puede explicarse por la utilización de la intervención esterilizada en los mercados cambiarios que utiliza con frecuencia el Banco de México para mitigar fundamentalmente depreciaciones no deseadas (Mántey, 2009: 72), y así lograr oportunamente el cumplimiento de la meta de inflación.

A su vez los modelos EGARCH (modelos 7 y 8 del Cuadro 3 ) muestran que el proceso EGARCH fue significativo para el periodo de análisis en la mayoría de los casos para ambos países, utilizando un nivel de significancia del Z-estadístico mayor a dos en valor absoluto. Con relación al componente $\varepsilon_{\mathrm{it}-1} / \sigma_{\mathrm{it}-1}^{2}$, como es distinto de cero en ambos modelos (3y 4) tanto para México como para Brasil, nos afirma que, primero, los shocks son asimétricos, y luego, como es significativo y con signo positivo para México, implica que shocks positivos de depreciación cambiaria tienen un impacto mayor sobre la volatilidad cambiaria que shocks negativos de la misma magnitud. De hecho, para el caso de México, el efecto estimado de los shocks positivos en el presente modelo EGARCH tiene el mismo signo y es significativo al estimado en el modelo TGARCH, 0.35 vs 0.16, respectivamente. En esa línea, como es significativo y con signo negativo para Brasil, implica que shocks positivos de depreciación cambiaria tienen un impacto menor sobre la volatilidad cambiaria que shocks negativos de la misma magnitud. Y también para el caso de Brasil, el efecto estimado de los shocks positivos en el presente modelo EGARCH tiene el mismo signo y es significativo al estimado en el modelo TGARCH, -0.20 vs -0.23 .

Los resultados de los modelos TGARCH y EGARCH (modelos 5, 6, 7 y 8 del Cuadro 3) para México y Brasil podrían explicarse por el hecho de que en México se trata de mantener el tipo de cambio en una senda de apreciación, para el cumplimiento de la meta de inflación 
deseada, mediante la utilización de la intervención esterilizada en los mercados cambiarios. Por su lado, en Brasil se utiliza la política monetaria y cambiaria para evitar apreciaciones no deseadas que vayan a ir en desmedro de sus exportaciones y balanza comercial.

A su vez, los modelos markovianos Markov Switching del Cuadro 7 permiten detectar regímenes de alta y baja volatilidad cambiaria. En particular sellega a las siguientes conclusiones. Primero, según el coeficiente sigma de ambos regímenes para ambos países, el tipo de cambio nominal de venta, tanto en México como en Brasil, es más volátil en periodos de alta volatilidad que en periodos de baja volatilidad. Segundo, los cuadros 8 y 9 indican respectivamente que los periodos de baja volatilidad en México y Brasil, en el periodo considerado, fueron relativamente persistentes con relación a los periodos de alta volatilidad. Este resultado nos llevaría inmediatamente a la conclusión de que en ambos países se han utilizado instrumentos monetarios para mantener controlado el tipo de cambio en una determinada dirección, de tal forma que la misma no ponga en peligro el cumplimiento de ciertos objetivos, entre ellos una meta de inflación deseada; asimismo, este resultado reflejaría que todavía persiste el denominado "miedo a flotar". Tercero, los cuadros 8 y 9 del modelo Markov Switching Autorregresivo MSAR (2) nos confirma, por ejemplo para México, que la probabilidad de pasar de un periodo de baja volatilidad a una de alta volatilidad es aproximadamente de 0.020; y que este resultado es menor que la probabilidad de pasar de un periodo de alta volatilidad a una de baja volatilidad (0.16). Por lo tanto, al observar la matriz de probabilidades de transición que se muestra en los cuadro 8 y 9 , se puede decir que, una vez que la volatilidad del tipo de cambio peso-dólar se encuentra en un estado o régimen, es muy baja la probabilidad de que pase al otro estado. En particular, se observa que, cuando se encuentra en el estado de baja volatilidad, es muy poco probable que pase al estado de alta volatilidad, siendo la probabilidad apenas superior a $2 \%$. Es relativamente mayor la probabilidad de que, estando la volatilidad en el régimen de volatilidad alta, pase al siguiente día al régimen de volatilidad baja (poco más de 16.5\%). Para el caso brasileño, el análisis es similar, y se puede advertir que también es relativamente mayor la probabilidad de que, estando la volatilidad en el régimen de volatilidad alta, pase al siguiente día al régimen de volatilidad baja (aproximadamente 12\%).

Por último, se puede advertir que la persistencia estimada de la volatilidad cambiaria pesodólar para México es del orden 0.8159; y para el real-dólar en Brasil, de 0.8585. Ello implica una persistencia alta en volatilidad, lo que confirma que los choques en ella no se disipan rápidamente, haciendo más riesgosa en términos del dólar la posición de un inversionista que se mantiene en moneda nacional. Este resultado explicaría en gran medida el proceso 
de extranjerización de ambas economías latinoamericanas, haciendo más atractivo y menos riesgoso invertir en dólares, así como la posibilidad de mantener los ahorros de las economías domésticas en moneda extranjera, todo ello debido al no abandono del ancla cambiaria hasta el día de hoy y al denominado "miedo a flotar" de ambas economías.

Fecha de recepción: 25 de febrero de 2016. Fecha de aceptación: 2 de mayo de 2016. Manejado por la A.B.C.E. 


\section{Referencias}

1. Andersen, T.G. y T. Bollerslev. 1998. "DM-Dollar Volatility: Intraday Activity Patterns, Macroeconomic Announcements and Longer-Run Dependencies", Journal of Finance, $53,2190-265$.

2. Awartani, B.M.A. y V. Corradi. 2005. "Predicting the volatility of the S\&P-500 stock index via GARCH models: the role of asymmetries", International Journal of Forecasting, 21, 167-183.

3. Banxico. 1993. Banco de México. Información económica.

4. Banxico. 2008. Banco de México. Información económica.

5. Benavides, G. y C. Capistrán. 2009. "Una nota sobre las volatilidades de la tasa de interés y del tipo de cambio según diferentes instrumentos de política monetaria: México, 1998-2008”. Banco de México, Documento de investigación, N²009-10.

6. Bollerslev, T. 1986. "Generalized Autoregressive Conditional Heteroskedasticity", Journal of Econometrics, 31, 307-327.

7. Bollerslev, T., R. Y. Chou y K. F. Kroner. 1992. "ARCH modeling in finance: A review of the theory and empirical evidence", Journal of econometrics, 52 (1-2), 5-59.

8. Bollerslev, T., R. F. Engle y D. B. Nelson. 1994. "ARCH models". Handbook of econometrics, 4, 2959-3038.

9. Baqueiro, A., A. Díaz de León y A. Torres García. 2003. “'Temor a la flotación o a la inflación? La importancia del 'traspaso' del tipo de cambio a los precios". Banco de México, Documento de investigación, $\mathrm{N}^{\circ} 02$.

10. Brooks, C. 2002. Introductory econometrics for finance. Cambridge University Press.

11. Caballero, Benigno. 2003. "Notas de clase de econometría y estadística”, Universidad Técnica de Oruro.

12. De Gregorio, J., A. Tokman y R. Valdés. 2005. "Flexible exchange rate with inflation targeting in Chile: Experience and issues", Inter-American Development Bank, Working Paper N540.

13. Diebold, F.X., J.H. Lee y G.C. Weinbach. 1993. "Regime -Switching with time varying transition probabilities". En: C. Hargreaves, Nonstationary Time Series and Cointegration. Oxford University Press. 
14. Diebold, F. X. y R. S. Mariano. 1995. "Comparing Predictive Accuracy”, Journal of Business and Economic Statistics, 13, 253-263.

15. Ding, Z. y C. W. Granger. 1996. "Modeling volatility persistence of speculative returns: a new approach", Journal of econometrics, 73 (1), 185-215.

16. Domowitz, I. y C. Hakkio. 1985. "Conditional Variance and the Risk Premium in the Foreign Exchange Market”, Journal of International Economics, vol. 19, 47-66.

17. Edwards, S. 2007. "The relationship between exchange rates and inflation targeting revisited". En: F. Mishkin y K. Schmidt-Hebbel (eds.), Monetary Policy under Inflation Targeting. Banco Central de Chile, Santiago de Chile.

18. Engel, C. y C. S. Hakkio. 1996. "The distribution of exchange rates in the EMS", International Journal of Finance \& Economics, 1 (1), 55-67.

19. Engle, R.F. 1982. "Autoregressive Conditional Heteroskedasticity with Estimates of Variance of U.K. Inflation", Econometrica, 50, 987-1007.

20. Engle, R y T. Bollerslev. 1986. "Modelling the Persistence of Conditional Variance", Econometric Review 5, 1-50 y 80-87.

21. Glosten, L.R., R. Jagannathan y D.E. Runkle. 1993. "On the relation between the expected value and the volatility of the nominal excess return on stocks", Journal of Finance, 48, 1779-801.

22. Gomez-Puig, M. y J. Montalvo. 1997. "A New Indicator to Assess the Credibility of the EMS”, European Economic Review, 41(8), 1511-1535.

23. Hamilton, J. 1989. "A New Approach to the Economic Analysis of Nonstationary Time Series and the Business Cycle", Econometrica, 57:357 -384.

24. -------- 1990. "Analysis of Time Series Subject to Changes in Regime", Journal of Econometrics, $45,39-70$.

25. ------- 1994. Times Series Analysis. Princenton University Press.

26. Hsieh, D. A. 1989. "Modeling Heterocedasticity in Daily Foreign-Exchange Rates: 1974-1983", Journal of Business and Economic Statistics, vol. 7, 307-17.

27. Lee, B. S. 1991. "Simulation estimation of time-series models", Journal of Econometrics, 47 (2-3), 197-205. 
28. McMillan, D.G. y A.E. Speight. 2006. "Long memory and heterogeneous components in high frequency pacific-basin exchange rate volatility”, Asia-Pacific Financial Markets, $\mathrm{N}^{\circ} 12,199-226$.

29. Malliaropulos, D. 1995. "Conditional Volatility of Exchange Rates and Risk Premia in the EMS”, Applied Economics, vol. 27, 117-123.

30. Mántey, Guadalupe. 2006. "Inflation Targeting and Exchange Rate Risk in Emerging Economies Subject to Structural Inflation”. En: S. Motames-Samadian (ed.), Economic and Financial Developments in Latin America. Londres: Palgrave Macmillan.

31. ------- 2009. "Intervención esterilizada en el mercado de cambios en un régimen de metas de inflación: la experiencia de México", Investigación económica, vol. 68, número especial, pp. 47-78.

32. Mohnot, R. 2011. "Forecasting Forex volatility in turbulent times", Global Journal of Business Research, 5 (1), 27-38.

33. Nelson, D.B. 1991. "Condicional Heteroskedasticity in Asset Returns: A New Approach", Econometrica, 59 (2), 347-70.

34. Olowe, R.A. 2009. "Modelling naira/dollar exchange rate volatility: application of GARCH and assymetric models”, International Review of Business Research Papers, 5 (3), 377-398.

35. Sánchez, A.y O. Reyes. 2006. "Regularidades probabilísticas de las series financieras y la familia de modelos GARCH”, Ciencia Ergo Sum, 13 (2), 149-156.

36. Sandoval, J. 2006. "Do asymmetric GARCH models fit better exchange rate volatilities on emerging markets?”, Odeon, $\mathrm{N}^{\circ}$ 3, 97-118.

37. Schwert, W. 1989. "Stock Volatility and Crash of '87”, Review of Financial Studies, 3, 77-102.

38. Sengupta, J.K. y R.E. Sfeir. 1996. "Modelling exchange rate volatility". Department of Economics, University of California in Santa Barbara y School of Business, Chapman University, Working Paper.

39. Taylor, S. 1986. Modelling Financial Time Series. New York: John Wiley \& Sons.

40. Tse, Y.K. 1998. "The conditional heteroscedasticity of the yen-dollar exchange rate", Journal of Applied Econometrics, 13 (1), 49-55.

41. Zakoian, J.M. 1994. "Threshold Heteroskedastic Models", Journal of Economic Dynamics and Control, 18, 931-944. 